\title{
SPECTRAL APPROXIMATION FOR SEGAL-BARGMANN SPACE TOEPLITZ OPERATORS
}

\author{
ALBRECHT BÖTTCHER and HARTMUT WOLF \\ Fakultät für Mathematik, TU Chemnitz-Zwickau \\ 09107 Chemnitz, Germany \\ E-mail: aboettch@mathematik.tu-chemnitz.de
}

\begin{abstract}
Let $A$ stand for a Toeplitz operator with a continuous symbol on the Bergman space of the polydisk $\mathbb{D}^{N}$ or on the Segal-Bargmann space over $\mathbb{C}^{N}$. Even in the case $N=1$, the spectrum $\Lambda(A)$ of $A$ is available only in a few very special situations. One approach to gaining information about this spectrum is based on replacing $A$ by a large "finite section", that is, by the compression $A_{n}$ of $A$ to the linear span of the monomials $\left\{z_{1}^{k_{1}} \ldots z_{N}^{k_{N}}: 0 \leq k_{j} \leq n\right\}$. Unfortunately, in general the spectrum of $A_{n}$ does not mimic the spectrum of $A$ as $n$ goes to infinity.

However, in the same way as in numerical analysis the question "Is $A$ invertible?" is replaced by the question "What is $\left\|A^{-1}\right\|$ ?", it turns out that the mysteries of $\Lambda\left(A_{n}\right)$ for large $n$ may be much better understood by considering the pseudospectrum of $A_{n}$ rather than the usual spectrum. For $\varepsilon>0$, the $\varepsilon$-pseudospectrum of an operator $T$ is defined as the set $\Lambda_{\varepsilon}(T)=\{\lambda \in$ $\left.\mathbb{C}:\left\|(T-\lambda I)^{-1}\right\| \geq 1 / \varepsilon\right\}$. Our central result says that the $\operatorname{limit}_{\lim } \rightarrow \infty\left\|A_{n}^{-1}\right\|$ exists and is equal to the maximum of $\left\|A^{-1}\right\|$ and the norms of the inverses of $2^{N}-1$ other operators associated with $A$. This result implies that for each $\varepsilon>0$ the $\varepsilon$-pseudospectrum of $A_{n}$ approaches the union of the $\varepsilon$-pseudospectra of $A$ and the $2^{N}-1$ operators associated with $A$. If in particular $N=1$, it follows that

$$
\Lambda(A)=\lim _{\varepsilon \rightarrow 0} \lim _{n \rightarrow \infty} \Lambda_{\varepsilon}\left(A_{n}\right),
$$

whereas, as already said, the equality $\Lambda(A)=\lim _{n \rightarrow \infty} \lim _{\varepsilon \rightarrow 0} \Lambda_{\varepsilon}\left(A_{n}\right)\left(=\lim _{n \rightarrow \infty} \Lambda\left(A_{n}\right)\right)$ is in general not true.

The paper does not aim at completeness, its purpose is rather to outline the ideas behind the theory, and especially, to illustrate the power of $C^{*}$-algebra techniques for tackling the problem of spectral approximation. We therefore focus our attention on Segal-Bargmann space Toeplitz operators. Our main theorems include Fredholm criteria for such operators, results on the norms
\end{abstract}

1991 Mathematics Subject Classification: Primary 47B35; Secondary 15A60, 30E10, 32A37, 45H05, 47N40.

Research of the first author supported by the Alfried Krupp Förderpreis für junge Hochschullehrer of the Krupp Foundation.

The paper is in final form and no version of it will be published elsewhere. 
of the inverses of their large truncations, as well as the foundation of several approximation methods for solving equations with a Segal-Bargmann space Toeplitz operator.

1. Introduction. The Segal-Bargmann space $A^{2}(\mathbb{C}, d \mu)$ is the Hilbert space of all entire functions $f$ on $\mathbb{C}$ satisfying

$$
\|f\|^{2}:=\int_{\mathbb{C}}|f(z)|^{2} d \mu(z)<\infty
$$

where $d \mu(z)=(1 / 2 \pi) e^{-|z|^{2} / 2} d A(z)$ and $d A(z)$ is area measure on $\mathbb{C}$, i.e. $d A(z)=r d r d \theta$ in polar coordinates. The space $A^{2}(\mathbb{C}, d \mu)$ has the reproducing kernel $e^{z \bar{w} / 2}$. This means that if a function $f(w)$ in $A^{2}(\mathbb{C}, d \mu)$ is multiplied by $e^{z \bar{w} / 2}$ and then integrated against $d \mu(w)$ over $\mathbb{C}$, we get back $f(z)$ :

$$
\int_{\mathbb{C}} e^{z \bar{w} / 2} f(w) d \mu(w)=f(z) .
$$

Now choose a function $a(w)$ on $\mathbb{C}$, multiply $f(w)$ by $a(w) e^{z \bar{w} / 2}$ and integrate against $d \mu(w)$ over $\mathbb{C}$; what results is in general a new function $g(z)$ :

$$
\int_{\mathbb{C}} a(w) e^{z \bar{w} / 2} f(w) d A(w)=g(z) .
$$

The subject of this paper is the integral equation (2), that is, we think of $a(w)$ and $g(z)$ as given functions and are looking for a function $f(w)$ which satisfies (2).

The Segal-Bargmann space was introduced by Segal [23] and Bargmann [2] in the early sixties. It turns out that representations of the Heisenberg group on the Segal-Bargmann space have particularly interesting properties (see e.g. [16]). Problems in quantum mechanics lead to kernels $a(w) e^{z \bar{w} / 2}$ in $(2)$ for which $a(w)$ shows highly complicated behavior at infinity. For example, anti-Wick quantization results in the case where $a(w)=p(\bar{w}, w)$ and $p$ is a polynomial of two variables (see [16]); in this situation $a(w)$ is unbounded. Or, when using Weyl operators to represent the canonical commutation relations on $\mathbb{C}$, we encounter equation (2) with $a(w)=e^{i \operatorname{Re}(\alpha w)}$, that is, with an almost periodic function $a(w)$ (see [3] and [4]).

At present time one is not yet able to tackle equation (2) effectively if $a(w)$ is unbounded or almost periodic, and no progress in these two directions will be made in this paper. A Fredholm theory for equations of the form (2) has been worked out in the cases where $a(w)$ is uniformly continuous on $\mathbb{C}$ or where $a(w)$ oscillates at infinity "less than linearly" by Berger and Coburn in [3] and [4]. Several methods for approximately solving equations like (2) have been studied by the authors (see [5], [9], [11], [12]) for uniformly continuous functions $a(w)$. Notice that in the latter case the limit

$$
a_{\infty}\left(e^{i \theta}\right):=\lim _{r \rightarrow \infty} a\left(r e^{i \theta}\right)
$$

exists for every $e^{i \theta}$ on the complex unit circle $\mathbb{T}$ and that $a_{\infty}$ is a continuous function on $\mathbb{T}$; hence $a(w)$ behaves very well at infinity.

This paper deals with the case where $a(w)$ is piecewise continuous (in a sense that will be specified in the following). For instance, if $a(w)=b(w)$ for $\operatorname{Re} w>0$ and $a(w)=c(w)$ for $\operatorname{Re} w<0$, where $b(w)$ and $c(w)$ are uniformly continuous, then $a(w)$ is piecewise continuous in our sense. For the functions $a(w)$ we consider here the limits $(3)$ exist for 
almost all $e^{i \theta} \in \mathbb{T}$, but the function $a_{\infty}$, which describes the behavior of $a(w)$ at infinity, is now a piecewise continuous function on $\mathbb{T}$.

Our main results provide a Fredholm theory of equation (2) and its higher-dimensional analogues and prove the convergence of certain approximation methods for such equations. We also establish results on the norms of the inverses and on the pseudospectra of truncations of the operator given by the left-hand side of (2).

2. Fredholm results. The Segal-Bargmann space $A^{2}(\mathbb{C}, d \mu)$ is a closed subspace of the Hilbert space $L^{2}(\mathbb{C}, d \mu)$, and the orthogonal projection $P$ of $L^{2}(\mathbb{C}, d \mu)$ onto the space $A^{2}(\mathbb{C}, d \mu)$ is given by

$$
(P f)(z)=\int_{\mathbb{C}} e^{z \bar{w} / 2} f(w) d \mu(w) \quad\left(f \in L^{2}(\mathbb{C}, d \mu)\right) .
$$

With this expression for $P$, equality (1) amounts to the trivial fact that $P f=f$ for all $f \in A^{2}(\mathbb{C}, d \mu)$. An orthonormal basis $\left\{e_{n}\right\}_{n=0}^{\infty}$ in $A^{2}(\mathbb{C}, d \mu)$ is constituted by the functions

$$
e_{n}(z)=\left(2^{n} n !\right)^{-1 / 2} z^{n}
$$

Each function $a \in L^{\infty}(\mathbb{D})$ induces a bounded operator $T^{1}(a)$ on the space $A^{2}(\mathbb{C}, d \mu)$ by the formula $T^{1}(a) f=P(a f)$. This operator is called a Toeplitz operator and the function $a$ is referred to as the symbol of $T^{1}(a)$. We remark that $T^{1}(a)$ acts by the rule

$$
\left(T^{1}(a) f\right)(z)=\int_{\mathbb{C}} a(w) e^{z \bar{w} / 2} f(w) d \mu(w) \quad(z \in \mathbb{C}),
$$

so that equation (2) is nothing but the equation $T^{1}(a) f=g$. For an operator $A$ on $A^{2}(\mathbb{C}, d \mu)$, we denote by $A_{j k}(j, k \geq 0)$ the $j k$ entry of the matrix representation of $A$ in the orthonormal basis $\left\{e_{n}\right\}$ given by (4). A straightforward computation shows that

$$
\left[T^{1}(a)\right]_{j k}=\frac{1}{\sqrt{2^{j} j ! 2^{k} k !}} \int_{\mathbb{C}} a(w) w^{k} \bar{w}^{j} d \mu(w) .
$$

Suppose now that $a \in L^{\infty}(\mathbb{C})$ is a function for which the limits (3) exist for almost all $e^{i \theta} \in \mathbb{T}$ and for which $a_{\infty}$ belongs to $L^{\infty}(\mathbb{T})$. We then put

$$
a_{n}=\frac{1}{2 \pi} \int_{0}^{2 \pi} a_{\infty}\left(e^{i \theta}\right) e^{-i n \theta} d \theta \quad(n \in \mathbb{Z})
$$

and we let $T^{0}(a)$ stand for the operator on $A^{2}(\mathbb{C}, d \mu)$ given by $\left[T^{0}(a)\right]_{j k}=a_{j-k}$. Since the Toeplitz matrix $\left(a_{j-k}\right)_{j, k=0}^{\infty}$ induces a bounded operator on $l^{2}\left(\mathbb{Z}_{+}\right)$whenever the entries $a_{j-k}$ are related to a function $a_{\infty} \in L^{\infty}(\mathbb{T})$ via (6), it follows that $T^{0}(a)$ is a well-defined bounded operator on $A^{2}(\mathbb{C}, d \mu)$. In fact, the operator $T^{0}(a)$ is unitarily equivalent to the Toeplitz operator with the symbol $a_{\infty}$ on the Hardy space $H^{2}(\mathbb{T})$, and the latter operators are fairly well understood (see e.g. [14] or [17]).

Let $\overline{\mathbb{C}}$ denote the maximal ideal space of the $C^{*}$-algebra of all continuous functions $a$ on $\mathbb{C}$ for which the limits (3) exist for all $e^{i \theta} \in \mathbb{T}$ and represent a continuous function $a_{\infty}$ on $\mathbb{T}$. Thus, $\overline{\mathbb{C}}$ is the compactification of $\mathbb{C}$ by a circle $\mathbb{T}_{\infty}=\left\{\infty e^{i \theta}: e^{i \theta} \in \mathbb{T}\right\}$ at infinity. A neighborhood base of $\infty e^{i \theta} \in \mathbb{T}$ is given by $\left\{U_{R}\left(\infty e^{i \theta}\right)\right\}_{R>0}$ with

$$
U_{R}\left(\infty e^{i \theta}\right)=\left\{r e^{i \phi} \in \overline{\mathbb{C}}: r \in(R, \infty), \phi \in(\theta-1 / R, \theta+1 / R)\right\} .
$$


With this notation, $C(\overline{\mathbb{C}})$ is the $C^{*}$-algebra of all uniformly continuous functions on $\mathbb{C}$, that is, the $C^{*}$-algebra of all continuous functions $a$ on $\mathbb{C}$ with the property that for every $\varepsilon>0$ there are an $R=R(\varepsilon)>0$ and a $\delta=\delta(\varepsilon)>0$ such that

$$
\left|a\left(r e^{i \theta}\right)-a\left(\rho e^{i \phi}\right)\right|<\varepsilon
$$

whenever $r, \rho>R$ and $|\theta-\phi|<\delta$.

If $a \in C(\overline{\mathbb{C}})$, then $T^{1}(a)-T^{0}(a)$ is compact. This has certainly been known for a long time (see [13] for the Bergman space case), but since we had not been able to find an explicit reference, we gave a proof in [12]. By having recourse to the well-elaborated theory of the Hardy space Toeplitz operators $T^{0}(a)$, one can so gain a lot of insights into the Segal-Bargmann space Toeplitz operators $T^{1}(a)$ with $a \in C(\overline{\mathbb{C}})$.

Let $\mathcal{L}$ and $\mathcal{K}$ be the bounded and compact operators on $A^{2}(\mathbb{C}, d \mu)$, respectively. We denote by $\mathcal{C}$ the smallest $C^{*}$-subalgebra of $\mathcal{L}$ containing the set $\left\{T^{1}(a): a \in C(\overline{\mathbb{C}})\right\} \cup \mathcal{K}$, put $\mathcal{C}^{\pi}=\mathcal{C} / \mathcal{K}$ and denote the coset $A+\mathcal{K}$ by $A^{\pi}$. Using the compactness of $T^{1}(a)-T^{0}(a)$ for $a \in C(\overline{\mathbb{C}})$ and the results of [14] and [17] (for example), one can easily establish the following.

Theorem 2.1. The $C^{*}$-algebra $\mathcal{C}^{\pi}$ is commutative, its maximal ideal space may be identified with the unit circle $\mathbb{T}$, every element of $\mathcal{C}^{\pi}$ is of the form $\left[T^{1}(a)\right]^{\pi}$ with some $a \in C(\overline{\mathbb{C}})$, and the mapping

$$
\gamma_{c}: \mathcal{C}^{\pi} \rightarrow C(\mathbb{T}), \quad\left[T^{1}(a)\right]^{\pi} \mapsto a_{\infty}
$$

is an isometric $C^{*}$-algebra isomorphism.

In particular, if $\left\{a_{j k}\right\}$ is a finite collection of functions in $C(\overline{\mathbb{C}})$, then the operator

$$
A=\sum_{j} \prod_{k} T^{1}\left(a_{j k}\right) \in \mathcal{C}
$$

is Fredholm (i.e. invertible modulo compact operators) if and only if

$$
\gamma_{c}\left(A^{\pi}\right)=\left(\sum_{j} \prod_{k} a_{j k}\right)_{\infty} \in C(\mathbb{T})
$$

does not vanish on $\mathbb{T}$; in that case the index (i.e. the difference of the kernel and cokernel dimensions) of $A$ equals minus the winding number of the curve traced out by $\gamma_{c}\left(A^{\pi}\right)$ with respect to the origin.

We now define what we mean by piecewise continuous functions on $\mathbb{C}$. Fix $\tau=e^{i \theta} \in \mathbb{T}$ (and thus $\infty \tau \in \mathbb{T}_{\infty}$ ), let $g_{0, \tau}$ denote the straight line through 0 and $\tau$ (oriented in such a way that 0 comes before $\tau$ ), and for $d \in \mathbb{R}$, let $g_{d, \tau}$ denote the (oriented) straight line which results from $g_{0, \tau}$ by translating it by the distance $|d|$ to the left $(d \geq 0)$ or to the right $(d<0)$. Hence, if $g_{0, \tau}$ is given by

$$
g_{0, \tau}=\left\{r e^{i \theta}:-\infty<r<\infty\right\}
$$

then $g_{d, \tau}$ is the straight line

$$
g_{d, \tau}=\left\{(i d+r) e^{i \theta}:-\infty<r<\infty\right\} .
$$


Given $a \in L^{\infty}(\mathbb{T})$, we write $a \in P C_{d, \tau}$ if there are two numbers, denoted by $a(\tau-0)$ and $a(\tau+0)$, such that

$$
\lim _{z \rightarrow \infty \tau, z \in H_{d, \tau}^{ \pm}} a(z)=a(\tau \pm 0),
$$

where $H_{d, \tau}^{+}$and $H_{d, \tau}^{-}$denote the half-planes lying on the left and right of $g_{d, \tau}$ respectively.

If $d: \mathbb{T} \rightarrow \mathbb{R}$ is a continuous function, we let $P C_{d}$ stand for the set of all functions $a \in L^{\infty}(\mathbb{C})$ belonging to $P C_{d(\tau), \tau}$ for every $\tau \in \mathbb{T}$. It is readily seen that $P C_{d}$ is in fact a $C^{*}$-subalgebra of $L^{\infty}(\mathbb{C})$. From now on we leave the function $d: \mathbb{T} \rightarrow \mathbb{R}$ fixed and call the functions in $P C_{d}$ piecewise continuous.

Any finite collection $\left\{g_{j}\right\}$ of straight lines divides the plane $\mathbb{C}$ into a finite number of connected components $E_{k}$ :

$$
\bigcup_{k} E_{k}=\mathbb{C} \backslash \bigcup_{j} g_{j}
$$

If no two of the lines $g_{j}$ are parallel, then every function $a \in L^{\infty}(\mathbb{C})$ whose restriction to each of the components $E_{k}$ is uniformly continuous belongs to $P C_{d}$ for some $d: \mathbb{T} \rightarrow \mathbb{R}$. On the other hand, such functions as

$$
a(z)= \begin{cases}1 & \text { for } 0<\operatorname{Re} z<1, \\ 0 & \text { for } \operatorname{Re} z<0 \text { or } \operatorname{Re} z>1,\end{cases}
$$

are not in $P C_{d}$ for any $d$ and are therefore not piecewise continuous in our sense.

If $a \in P C_{d}$, then the limits (3) exist for almost all $e^{i \theta} \in \mathbb{T}$ and represent a piecewise continuous function $a_{\infty}$ on $\mathbb{T}$, i.e. a function $a_{\infty} \in L^{\infty}(\mathbb{T})$ such that the one-sided limits $a\left(e^{i(\theta \pm 0)}\right)$ exist for every $e^{i \theta} \in \mathbb{T}$. It is well-known that piecewise continuous functions on $\mathbb{T}$ may have at most countably many jumps, so that $a\left(e^{i(\theta-0)}\right)=a\left(e^{i(\theta+0)}\right)$ for all but countably many points $e^{i \theta} \in \mathbb{T}$. The main difficulty with piecewise continuous symbols is that $T^{1}(a)-T^{0}(a)$ is in general not compact if $a \in P C_{d}$.

Let $\mathcal{B}$ denote the smallest $C^{*}$-subalgebra of $\mathcal{L}$ containing all operators $T^{1}(a)$ with $a \in P C_{d}$ and all compact operators. To study the quotient algebra $\mathcal{B}^{\pi}=\mathcal{B} / \mathcal{K}$, we make use of the fact that

$$
T^{1}(a) T^{1}(c)-T^{1}(a c) \quad \text { and } \quad T^{1}(c) T^{1}(a)-T^{1}(c a)
$$

are compact whenever $a \in L^{\infty}(\mathbb{C})$ and $c \in C(\overline{\mathbb{C}})$ (see [4]). Thus, $\mathcal{C}^{\pi}$ is contained in the center of $\mathcal{B}^{\pi}$, and we may so have recourse to the following result, which is known as the local principle of Allan [1] and Douglas [14].

TheOREm 2.2. Let $B$ be a $C^{*}$-algebra with unit element and let $C$ be a $C^{*}$-subalgebra of the center of $B$ containing the unit element. Denote the maximal ideal space of $C$ by $M(C)$, and for $m \in M(C)$, let $\mathcal{Y}_{m}$ be the smallest closed two-sided star-ideal of $B$ containing $m$. Then $\bigcap_{m \in M(C)} \mathcal{Y}_{m}=\{0\}$, the mapping

$$
\Gamma: B \rightarrow \bigoplus_{m \in M(C)} B / \mathcal{Y}_{m}, \quad b \mapsto\left(b+\mathcal{Y}_{m}\right)_{m \in M(C)}
$$

is an isometric $C^{*}$-algebra homomorphism, and in particular, an element $b \in B$ is invertible in $B$ if and only if $b+\mathcal{Y}_{m}$ is invertible in $B / \mathcal{Y}_{m}$ for every $m \in M(C)$. 
From Theorem 2.1 we know that $M\left(\mathcal{C}^{\pi}\right)$ may be identified with $\mathbb{T}$ : the maximal ideal of $\mathcal{C}^{\pi}$ corresponding to $\tau \in \mathbb{T}$ is given by

$$
\left\{\left[T^{1}(c)\right]^{\pi}: c \in C(\overline{\mathbb{C}}), c_{\infty}(\tau)=0\right\} .
$$

The smallest closed two-sided star-ideal $\mathcal{Y}_{\tau}$ of $\mathcal{B}^{\pi}$ containing this ideal equals

$$
\left\{\left[T^{1}(a)\right]^{\pi}: a \in P C_{d}, a_{\infty} \text { is continuous at } \tau, a_{\infty}(\tau)=0\right\} .
$$

Now let $a \in P C_{d}$. Denote by $\chi$ the characteristic function of the half-plane $H_{d(\tau), \tau}^{+}$. It is easily seen that

$$
\left[T^{1}(a)\right]^{\pi}+\mathcal{Y}_{\tau}=\left[T^{1}(a(\tau+0) \chi+a(\tau-0)(1-\chi))\right]^{\pi}+\mathcal{Y}_{\tau} .
$$

Since $\mathcal{B}^{\pi}$ is generated by all $\left[T^{1}(a)\right]^{\pi}$ with $a \in P C_{d}$, it follows that $\mathcal{B}^{\pi} / \mathcal{Y}_{\tau}$ is the $C^{*}$-algebra generated by the identity element and $\left[T^{1}(\chi)\right]^{\pi}+\mathcal{Y}_{\tau}$. One can show as in [10], p. 135 that the spectrum of $\left[T^{1}(\chi)\right]^{\pi}+\mathcal{Y}_{\tau}$ is the interval $[0,1]$, and hence $\mathcal{B}^{\pi} / \mathcal{Y}_{\tau}$ is isometrically isomorphic to $C([0,1])$. Now Theorem 2.2 gives the following

THEOREM 2.3. The $C^{*}$-algebra $\mathcal{B}^{\pi}$ is commutative, its maximal ideal space may be identified with the cylinder $\mathbb{T} \times[0,1]$ (with an exotic topology), and the Gelfand transform $\gamma_{b}$ of $\mathcal{B}^{\pi}$ onto $C(\mathbb{T} \times[0,1])$ is for $a \in P C_{d}$ given by

$$
\left(\gamma_{b}\left[T^{1}(a)\right]^{\pi}\right)(\tau, \mu)=a_{\infty}(\tau-0)(1-\mu)+a_{\infty}(\tau+0) \mu .
$$

For $a \in P C_{d}$, denote by $a^{\#}$ the closed, continuous, and naturally oriented curve resulting from the (essential) range of $a_{\infty}$ by filling in a line segment between the endpoints of each jump. The following theorem can be obtained from Theorem 2.3 by standard arguments (see e.g. [14] or [17]).

ThEOREM 2.4. Let $a \in P C_{d}$. Then $T^{1}(a)$ is Fredholm if and only if $a^{\#}$ does not contain the origin. In that case the index of $T^{1}(a)$ is minus the winding number of a \# about the origin.

For Hardy space Toeplitz operators the above two theorems are due to Gohberg and Krupnik [18] (but also see Widom [25]), for Bergman space Toeplitz operators they were first established by Gerard McDonald [20].

3. Finite section method. The finite section method for approximately solving equation (2) consists in the following. We look for an approximate solution $f_{n}$ in the form of a polynomial of degree at most $n$, i.e. in the form of a linear combination

$$
f_{n}(w)=c_{0}^{(n)} e_{0}(w)+\ldots+c_{n}^{(n)} e_{n}(w) \quad(w \in \mathbb{C})
$$

where the functions $e_{n}$ are given by (4), and the $n+1$ unknown coefficients $c_{0}^{(n)}, \ldots, c_{n}^{(n)}$ are determined so that the first $n+1$ Taylor coefficients at the origin of $T^{1}(a) f$ and $g$ coincide. Note that the equation $(2)$ is equivalent to an equation on $l^{2}\left(\mathbb{Z}_{+}\right)$with the infinite matrix $\left[T^{1}(a)\right]$ (whose entries are written down in (5)) and that the finite section method amounts to replacing this matrix by its principal $(n+1) \times(n+1)$-section.

Denote by $P_{n}(n=0,1,2, \ldots)$ the orthogonal projection of $A^{2}(\mathbb{C}, d \mu)$ onto the linear hull of $\left\{e_{0}, \ldots, e_{n}\right\}$, and let $T_{n}^{1}(a)$ stand for the compression of $T^{1}(a)$ onto the image $\operatorname{Im} P_{n}$ :

$$
T_{n}^{1}(a)=P_{n} T^{1}(a) \mid \operatorname{Im} P_{n}
$$


The finite section method replaces the equation $T^{1}(a) f=g$ by the equations

$$
T_{n}^{1}(a) f_{n}=P_{n} g \quad\left(f_{n} \in \operatorname{Im} P_{n}\right) .
$$

We say that the finite section method is applicable to $T^{1}(a)$ if there is an $n_{0} \geq 0$ such that the equations (8) are uniquely solvable for all $n \geq n_{0}$ and all $g \in A^{2}(\mathbb{C}, d \mu)$ and the functions $f_{n}$ converge in $A^{2}(\mathbb{C}, d \mu)$ to a solution $f \in A^{2}(\mathbb{C}, d \mu)$ of the equation $T^{1}(a) f=g$.

The finite section method for Hardy space Toeplitz operators and their compact perturbations has been studied for a long time and these investigations have now resulted in a round theory (see [17] and [8]). Taking into account that $T^{1}(a)=T^{0}(a)+K$ with some compact operator $K$ whenever $a \in C(\overline{\mathbb{C}})$, one so can easily establish the following result (see [9] and [12]).

THEOREM 3.1. Let $a \in C(\overline{\mathbb{C}})$. Then the finite section method is applicable to $T^{1}(a)$ if and only if $T^{1}(a)$ is invertible.

We will prove that the conclusion of the preceding theorem also holds for $a \in P C_{d}$. Since in that case the difference $T^{1}(a)-T^{0}(a)$ is in general not compact, we employ the algebraic approach developed in [24] and [7].

Let $\mathfrak{F}$ denote the collection of all sequences $\left\{A_{n}\right\}_{n=0}^{\infty}$ of operators $A_{n} \in \mathcal{L}\left(\operatorname{Im} P_{n}\right)$ such that

$$
\left\|\left\{A_{n}\right\}\right\|:=\sup _{n \geq 0}\left\|A_{n}\right\|<\infty .
$$

With obvious algebraic operations and the norm $(9)$, the set $\mathfrak{F}$ is a $C^{*}$-algebra. Let $\mathfrak{N}$ be the subset of $\mathfrak{F}$ consisting of all sequences $\left\{A_{n}\right\}$ with $\left\|A_{n}\right\| \rightarrow 0$ as $n \rightarrow \infty$. Clearly, $\mathfrak{N}$ is a closed two-sided star-ideal of $\mathfrak{F}$, and we may so consider the quotient algebra $\mathfrak{F} / \mathfrak{N}$. Note that if $\left\{A_{n}\right\} \in \mathfrak{F}$, then

$$
\left\|\left\{A_{n}\right\}+\mathfrak{N}\right\|=\limsup _{n \rightarrow \infty}\left\|A_{n}\right\|
$$

A sequence $\left\{A_{n}\right\} \in \mathfrak{F}$ is said to be stable if the operators $A_{n}$ are invertible for all sufficiently large $n$, for $n \geq n_{0}$ say, and if

$$
\sup _{n \geq n_{0}}\left\|A_{n}^{-1}\right\|<\infty .
$$

Equivalently, $\left\{A_{n}\right\} \in \mathfrak{F}$ is stable if and only if

$$
\limsup _{n \rightarrow \infty}\left\|A_{n}^{-1}\right\|<\infty
$$

where here and in what follows we put $\left\|A^{-1}\right\|=\infty$ if $A$ is not invertible. Hence, in algebraic language, the stability of $\left\{A_{n}\right\}$ is equivalent to the invertibility of $\left\{A_{n}\right\}+\mathfrak{N}$ in $\mathfrak{F} / \mathfrak{N}$. It is easy to see that the finite section method is applicable to $T^{1}(a)$ if and only if $T^{1}(a)$ is invertible and $\left\{T_{n}^{1}(a)\right\}$ is stable. Thus, we are led to studying the problem of the invertibility of $\left\{T_{n}^{1}(a)\right\}+\mathfrak{N}$ in $\mathfrak{F} / \mathfrak{N}$.

We will now show that if $a \in P C_{d}$ has at most finitely many jumps and if $c \in C(\overline{\mathbb{C}})$, then

$$
\begin{gathered}
T_{n}^{1}(a c)=T_{n}^{1}(a) T_{n}^{1}(c)+P_{n} K P_{n}+W_{n} L W_{n}+C_{n}, \\
T_{n}^{1}(c a)=T_{n}^{1}(c) T_{n}^{1}(a)+P_{n} M P_{n}+W_{n} N W_{n}+D_{n},
\end{gathered}
$$


where $K, L, M, N$ are compact, $\left\|C_{n}\right\| \rightarrow 0$ and $\left\|D_{n}\right\| \rightarrow 0$ as $n \rightarrow \infty$, and $W_{n}(n=$ $0,1,2, \ldots)$ are the operators which act on $A^{2}(\mathbb{C}, d \mu)$ by the rule

$$
W_{n}: \sum_{j=0}^{\infty} c_{j} e_{j} \mapsto c_{0} e_{n}+c_{1} e_{n-1}+\ldots+c_{n} e_{0},
$$

with $e_{n}$ given by (4). Formulas like (10), (11) were first established by Widom [26] for Hardy space Toeplitz operators and they govern computations with elements of the form $\left\{T_{n}^{1}(a)\right\}+\mathfrak{N}$ in $\mathfrak{F} / \mathfrak{N}$. The extension of Widom's formula to the Segal-Bargmann space case is not trivial.

Let $\sigma$ be the characteristic function of the half-plane

$$
\mathbb{C}_{\theta_{1}}:=\left\{z=r e^{i \theta}: r>0, \theta_{1}<\theta<\theta_{1}+\pi\right\} .
$$

Put $\theta_{2}=\theta_{1}+\pi$. A straightforward computation yields

$$
\begin{gathered}
{\left[T^{1}(\sigma)\right]_{j k}=\frac{\Gamma\left(\frac{k+j}{2}+1\right)}{\sqrt{\Gamma(k+1) \Gamma(j+1)}} \frac{e^{i \theta_{2}(k-j)}-e^{i \theta_{1}(k-j)}}{2 \pi i(k-j)} \quad(j \neq k),} \\
{\left[T^{1}(\sigma)\right]_{j j}=1 / 2,}
\end{gathered}
$$

and

$$
\begin{gathered}
{\left[T^{0}(\sigma)\right]_{j k}=\frac{e^{i \theta_{2}(k-j)}-e^{i \theta_{1}(k-j)}}{2 \pi i(k-j)} \quad(j \neq k),} \\
{\left[T^{0}(\sigma)\right]_{j j}=1 / 2 .}
\end{gathered}
$$

Now denote by $\rho$ the characteristic function of the strip

$$
S_{d, \theta_{1}}:=\left\{z \in \mathbb{C}: 0<\operatorname{Im}\left(z e^{-i \theta_{1}}\right)<d\right\} .
$$

We have

$$
\begin{aligned}
{\left[T^{1}(\rho)\right]_{j k} } & =\left(2^{k} k ! 2^{j} j !\right)^{-1 / 2} \int_{S_{d, \theta_{1}}} z^{k} \bar{z}^{j} d \mu(z) \\
& =\left(2^{k} k ! 2^{j} j !\right)^{-1 / 2} 2 \int_{0}^{\infty} r^{k+j+1} e^{-r^{2} / 2} d r \int_{\theta_{1}}^{\theta_{1}+\theta_{r}} e^{i(k-j) \theta} \frac{d \theta}{2 \pi},
\end{aligned}
$$

where $\theta_{r}=\pi / 2$ for $r \leq d$ and $\theta_{r}=\arcsin (d / r)$ for $r>d$. Obviously, $\theta_{r}=O(1 / r)$ as $r \rightarrow \infty$.

Lemma 3.2. For all $j, k$,

$$
\begin{aligned}
& \left|\left[T^{1}(\rho)\right]_{j k}\right| \leq D /|k-j| \quad(k \neq j), \\
& \left|\left[T^{1}(\rho)\right]_{j k}\right| \leq E \Gamma\left(\frac{k+j+1}{2}\right) / \sqrt{\Gamma(k+1) \Gamma(j+1)}
\end{aligned}
$$

with some constants $D$ and $E$.

Pro of. Since $\theta_{r}=O(1 / r)$, we get

$$
\begin{aligned}
\left|\left[T^{1}(\rho)\right]_{j k}\right| & \leq C\left(2^{k} k ! 2^{j} j !\right)^{-1 / 2} \int_{0}^{\infty} r^{k+j} e^{-r^{2} / 2} d r \\
& =C 2^{-1 / 2} \Gamma\left(\frac{k+j+1}{2}\right) / \sqrt{\Gamma(k+1) \Gamma(j+1)}
\end{aligned}
$$


and we thus obtain (16). If $k \neq j$ then

$$
\left|\int_{\theta_{1}}^{\theta_{1}+\theta_{r}} e^{i(k-j) \theta} d \theta\right|=\frac{\left|e^{i\left(\theta_{1}+\theta_{r}\right)(k-j)}-e^{i \theta_{1}(k-j)}\right|}{|k-j|} \leq \frac{2}{|k-j|},
$$

which gives

$$
\left|\left[T^{1}(\rho)\right]_{j k}\right| \leq \frac{E}{|k-j|} \Gamma\left(\frac{k+j+1}{2}\right) / \sqrt{\Gamma(k+1) \Gamma(j+1)},
$$

and because $\Gamma((x+y) / 2) \leq \sqrt{\Gamma(x) \Gamma(y)}$, we arrive at (17).

Lemma 3.3. Let $P_{n}$ and $W_{n}$ be as before and define $V^{n+1}(n=0,1,2, \ldots)$ by

$$
V^{n+1}: \sum_{j=0}^{\infty} c_{j} e_{j} \mapsto c_{0} e_{n+1}+c_{1} e_{n+2}+c_{2} e_{n+3}+\ldots
$$

Then the four operators

$$
\begin{array}{cc}
W_{n} T^{1}(\rho) W_{n}, & W_{n}\left(T^{1}(\sigma)-T^{0}(\sigma)\right) W_{n}, \\
P_{n} T^{1}(\rho) V^{n+1}, & P_{n}\left(T^{1}(\sigma)-T^{0}(\sigma)\right) V^{n+1}
\end{array}
$$

converge strongly to zero as $n \rightarrow \infty$.

Proof. Put $R=T^{1}(\sigma)-T^{0}(\sigma)$ and let $R_{j k}$ be the $j k$ entry of $R$. Then

$$
\left\|W_{n} R W_{n} e_{j}\right\|^{2}=\sum_{k=0}^{n}\left|R_{n-k, n-j}\right|^{2} .
$$

We first show that $W_{n} R W_{n} e_{j} \rightarrow 0$ as $n \rightarrow \infty$. Given any $\varepsilon>0$, choose an $N$ so that

$$
\sum_{k=N}^{\infty} 1 /|j-k|^{2}<\varepsilon / 2
$$

Stirling's formula gives

$$
\lim _{n \rightarrow \infty} \frac{\Gamma\left(n-\frac{k+j}{2}+1\right)}{\sqrt{\Gamma(n-k+1) \Gamma(n-j+1)}}=1,
$$

and hence there is an $n_{0}$ such that

$$
\left(1-\frac{\Gamma\left(n-\frac{k+j}{2}+1\right)}{\sqrt{\Gamma(n-k+1) \Gamma(n-j+1)}}\right)^{2}<\frac{\varepsilon}{2 N}
$$

for all $k=0,1, \ldots, N-1$ if only $n \geq n_{0}>N$. This together with (12) to (15) implies that if $n \geq n_{0}$, then

$$
\begin{aligned}
\sum_{k=0}^{n}\left|R_{n-k, n-j}\right|^{2} & =\sum_{k \in\{0, \ldots, n\} \backslash\{j\}}\left|R_{n-k, n-j}\right|^{2} \\
& \leq \frac{1}{\pi^{2}} \sum_{k \in\{0, \ldots, n\} \backslash\{j\}} \frac{1}{(j-k)^{2}}\left(1-\frac{\Gamma\left(n-\frac{k+j}{2}+1\right)}{\sqrt{\Gamma(n-k+1) \Gamma(n-j+1)}}\right)^{2} \\
& \leq \sum_{k \in\{0, \ldots, N-1\} \backslash\{j\}} \frac{1}{(j-k)^{2}} \frac{\varepsilon}{2 N}+\sum_{k=N}^{\infty} \frac{1}{(j-k)^{2}}<\varepsilon
\end{aligned}
$$


(recall that $\Gamma((x+y) / 2) / \sqrt{\Gamma(x) \Gamma(y)} \leq 1)$. Thus, $W_{n} R W_{n} e_{j} \rightarrow 0$ for all $j$, and since the operators $W_{n} R W_{n}$ are clearly uniformly bounded, it follows that $W_{n} R W_{n}$ converges strongly to zero as $n \rightarrow \infty$. In a similar way one can show that $P_{n} R V^{n+1} \rightarrow 0$ strongly as $n \rightarrow \infty$.

Let us now prove that $W_{n} T^{1}(\rho) W_{n} e_{j} \rightarrow 0$ as $n \rightarrow \infty$. Given any $\varepsilon>0$, define $N$ again by (18). By virtue of (17) and Stirling's formula,

$$
\left|\left[T^{1}(\rho)\right]_{n-k, n-j}\right| \leq \lim _{n \rightarrow \infty} \frac{E \Gamma\left(n-\frac{k+j}{2}+\frac{1}{2}\right)}{\sqrt{\Gamma(n-k+1) \Gamma(n-j+1)}}=0 .
$$

Hence, there is an $n_{0}$ such that

$$
\left|\left[T^{1}(\rho)\right]_{n-k, n-j}\right|^{2}<\varepsilon /(2 N)
$$

whenever $n \geq n_{0}>N$ and $0 \leq k \leq N-1$. So if $n \geq n_{0}$, we deduce from (16) that

$$
\sum_{k=0}^{n}\left|\left[T^{1}(\rho)\right]_{n-k, n-j}\right|^{2}<\sum_{k=0}^{N-1} \frac{\varepsilon}{2 N}+\sum_{k=N}^{\infty} \frac{D^{2}}{(j-k)^{2}}<\frac{\varepsilon}{2}+D^{2} \frac{\varepsilon}{2} .
$$

It now follows as above that $W_{n} T^{1}(\rho) W_{n} \rightarrow 0$ strongly as $n \rightarrow \infty$. One can show analogously that $P_{n} T^{1}(\rho) V^{n+1} \rightarrow 0$ as $n \rightarrow \infty$.

Lemma 3.4. Define the half-plane $H_{d, \tau}^{+}$as in Section 2 and let $\chi$ be the characteristic function of $H_{d, \tau}^{+}$. Then for every $c \in C(\overline{\mathbb{C}})$,

$$
\begin{aligned}
& T_{n}^{1}(\chi c)=T_{n}^{1}(\chi) T_{n}^{1}(c)+P_{n} M P_{n}+W_{n} N W_{n}+D_{n}, \\
& T_{n}^{1}(c \chi)=T_{n}^{1}(c) T_{n}^{1}(\chi)+P_{n} K P_{n}+W_{n} L W_{n}+C_{n},
\end{aligned}
$$

with compact operators $K, L, M, N$ and operators $C_{n}, D_{n}$ for which $\left\|C_{n}\right\|$ and $\left\|D_{n}\right\|$ converge to zero as $n \rightarrow \infty$.

Proof. We have $\chi=\sigma-\rho$ for suitably chosen $\theta_{1}$. The operator $K=T^{1}(\chi c)-$ $T^{1}(\chi) T^{1}(c)$ is known to be compact and clearly,

$$
T_{n}^{1}(\chi c)=T_{n}^{1}(\chi) T_{n}^{1}(c)+P_{n} T^{1}(\chi) Q_{n} T^{1}(c) P_{n}+P_{n} M P_{n}
$$

with $Q_{n}=I-P_{n}$. The operator $P_{n} T^{1}(\chi) Q_{n} T^{1}(c) P_{n}$ equals

$$
P_{n} T^{0}(\chi) Q_{n} T^{0}(c) P_{n}+P_{n} R(\chi) Q_{n} T^{0}(c) P_{n}+P_{n} T^{1}(\chi) Q_{n} R(c) P_{n},
$$

where $R(a):=T^{1}(a)-T^{0}(a)$. By Widom's original formula [26],

$$
P_{n} T^{0}(\chi) Q_{n} T^{0}(c) P_{n}=W_{n} L W_{n},
$$

where $L=T^{0}\left(\chi^{0} c^{0}\right)-T^{0}\left(\chi^{0}\right) T^{0}\left(c^{0}\right)$ and $a^{0}(z):=a(\bar{z})(z \in \mathbb{C})$. Again $L$ is known to be compact. Furthermore, since $R(c)$ is compact and $Q_{n}$ converges strongly to zero, it follows that

$$
\left\|P_{n} T^{1}(\chi) Q_{n} R(c) P_{n}\right\| \rightarrow 0 \quad \text { as } n \rightarrow \infty .
$$

Finally, from Formula 7.7(3) in [8] we infer that $Q_{n} T^{0}(c) P_{n}$ is equal to $V^{n+1} H W_{n}$ with some compact (Hankel) operator $H$. Due to Lemma 3.3, the operator $P_{n} R(\chi) V^{n+1}$ converges strongly to zero, and hence as $n \rightarrow \infty$,

$$
\left\|P_{n} R(\chi) Q_{n} T^{0}(c) P_{n}\right\|=\left\|P_{n} R(\chi) V^{n+1} H W_{n}\right\| \rightarrow 0 .
$$


This completes the proof of the first formula of the lemma. The second formula can be verified similarly.

Here now is the Segal-Bargmann space version of Widom's formula.

Proposition 3.5. Let $a \in P C_{d}$ and suppose $a_{\infty}$ has only a finite number of jumps. Then for every $c \in C(\overline{\mathbb{C}})$,

$$
\begin{aligned}
& T_{n}^{1}(a c)=T_{n}^{1}(a) T_{n}^{1}(c)+P_{n} M P_{n}+W_{n} N W_{n}+D_{n}, \\
& T_{n}^{1}(c a)=T_{n}^{1}(c) T_{n}^{1}(a)+P_{n} K P_{n}+W_{n} L W_{n}+C_{n},
\end{aligned}
$$

where $K, L, M, N$ are compact and $C_{n}, D_{n}$ converge uniformly to zero as $n$ goes to infinity.

Proof. Once Lemma 3.4 is available, the proof is the same as the one of Proposition 4.7 of $[10]$.

After having established the preceding proposition, the machinery worked out in $[7]$ and [8] for the Hardy space case and in [10] for the Bergman space operators can be carried over to the Segal-Bargmann space situation without difficulty.

Let $\mathfrak{J}$ denote the subset of $\mathfrak{F} / \mathfrak{N}$ consisting of all elements of the form $\left\{P_{n} K P_{n}+\right.$ $\left.W_{n} L W_{n}\right\}+\mathfrak{N}$ with compact operators $K$ and $L$. Define $\mathfrak{C}$ as the smallest $C^{*}$-subalgebra of $\mathfrak{F} / \mathfrak{N}$ containing $\mathfrak{J}$ and all elements $\left\{T_{n}^{1}(c)\right\}+\mathfrak{N}$ with $c \in C(\overline{\mathbb{C}})$, and let $\mathfrak{B}$ be the smallest $C^{*}$-subalgebra of $\mathfrak{F} / \mathfrak{N}$ containing $\mathfrak{J}$ and all elements $\left\{T_{n}^{1}(a)\right\}+\mathfrak{N}$ with $a \in P C_{d}$.

Proposition 3.6. Let $\left\{A_{n}\right\}+\mathfrak{N} \in \mathfrak{B}$. Then the two strong limits

$$
S_{1}\left\{A_{n}\right\}:=\operatorname{s-lim}_{n \rightarrow \infty} A_{n} \quad \text { and } \quad S_{2}\left\{A_{n}\right\}:=\operatorname{s-lim}_{n \rightarrow \infty} W_{n} A_{n} W_{n}
$$

exist and belong to $\mathcal{B}$. If even $\left\{A_{n}\right\}+\mathfrak{N} \in \mathfrak{C}$, then $S_{1}\left\{A_{n}\right\}$ and $S_{2}\left\{A_{n}\right\}$ lie in $\mathcal{C}$. If $a \in P C_{d}$, then

$$
S_{1}\left\{T_{n}^{1}(a)\right\}=T^{1}(a), S_{2}\left\{T_{n}^{1}(a)\right\}=T^{0}\left(a^{0}\right),
$$

where $a^{0}(z)=a(\bar{z})(z \in \mathbb{C})$, and if $A_{n}=P_{n} K P_{n}+W_{n} L W_{n}$ with $K, L \in \mathcal{K}$, then

$$
S_{1}\left\{A_{n}\right\}=K, S_{2}\left\{A_{n}\right\}=L .
$$

Pr oof. The same arguments as in the proof of Proposition 4.7 of [10] apply.

Using the previous proposition, it is readily seen that $\mathfrak{J}$ is a closed two-sided star ideal of both $\mathfrak{C}$ and $\mathfrak{B}$ (note, for example, that

$$
T_{n}^{1}(a) W_{n} L W_{n}=W_{n}\left(W_{n} T_{n}^{1}(a) W_{n}\right) L W_{n},
$$

and since $W_{n} T_{n}^{1}(a) W_{n}$ converges strongly to $T^{0}\left(a^{0}\right)$, it follows that

$$
T_{n}^{1}(a) W_{n} L W_{n}=W_{n} T_{n}^{0}\left(a^{0}\right) L W_{n}+C_{n}
$$

with $T^{0}\left(a^{0}\right) L \in \mathcal{K}$ and $\left.\left\{C_{n}\right\} \in \mathfrak{N}\right)$. Hence, we may consider the quotient algebras $\mathfrak{C}^{\pi}:=$ $\mathfrak{C} / \mathfrak{J}$ and $\mathfrak{B}^{\pi}:=\mathfrak{B} / \mathfrak{J}$. For $\left\{A_{n}\right\}+\mathfrak{N}$ in $\mathfrak{C}$ or $\mathfrak{B}$, we abbreviate $\left(\left\{A_{n}\right\}+\mathfrak{N}\right)+\mathfrak{J}$ to $\left\{A_{n}\right\}^{\pi}$.

Proposition 3.7. The mappings

$$
\delta_{c}: \mathfrak{C}^{\pi} \rightarrow \mathcal{C}^{\pi},\left\{A_{n}\right\}^{\pi} \mapsto\left(S_{1}\left\{A_{n}\right\}\right)^{\pi}
$$

and

$$
\delta_{b}: \mathfrak{B}^{\pi} \rightarrow \mathcal{B}^{\pi},\left\{A_{n}\right\}^{\pi} \mapsto\left(S_{1}\left\{A_{n}\right\}\right)^{\pi}
$$

are well-defined isometric $C^{*}$-algebra isomorphisms. 
The proof will be based on Theorem 2.2 and the following simple (well-known but extraordinarily useful) observation.

LEMMA 3.8. Let $B_{1}$ and $B_{2}$ be $C^{*}$-algebras and let $\delta: B_{1} \rightarrow B_{2}$ be a $C^{*}$-algebra homomorphism with the property that for every $b \in B_{1}$ the spectrum of $b$ coincides with the spectrum of $\delta(b)$. Then $\delta$ is an isometric $C^{*}$-algebra isomorphism of $B_{1}$ onto $\delta\left(B_{1}\right)$.

Proof of Lemma 3.8. Let $b \in B_{1}$. Then $\|b\|^{2}=\left\|b^{*} b\right\|$, and since $b^{*} b$ is self-adjoint, the norm $\left\|b^{*} b\right\|$ equals the spectral radius $r\left(b^{*} b\right)$. By assumption, $r\left(b^{*} b\right)$ coincides with $r\left(\delta\left(b^{*} b\right)\right)=r\left(\delta(b)^{*} \delta(b)\right)$ and hence, by the self-adjointness of $\delta(b)^{*} \delta(b)$, with $\left\|\delta(b)^{*} \delta(b)\right\|=$ $\|\delta(b)\|^{2}$.

The Hardy space analogue of Proposition 3.7 was established in [7]. Also see Theorem 7.33(b) of [8] for piecewise continuous symbols. Since $T^{1}(c)-T^{0}(c)$ is compact for $c \in$ $C(\overline{\mathbb{C}})$, the assertion on $\delta_{c}$ follows immediately from its Hardy space counterpart.

Proof of Proposition 3.7. Let us now prove the assertion for $\delta_{b}$. We first show that $\delta_{b}$ is well-defined. So let $\left\{A_{n}\right\}^{\pi}=0$, i.e. assume $\left\{A_{n}\right\}+\mathfrak{N} \in \mathfrak{J}$. Then $A_{n}=$ $P_{n} K P_{n}+W_{n} L W_{n}+C_{n}$ with $K, L \in \mathcal{K}$ and $\left\{C_{n}\right\} \in \mathfrak{N}$. It follows that $S_{1}\left\{A_{n}\right\}=K \in \mathcal{K}$ and hence $\left(S_{1}\left\{A_{n}\right\}\right)^{\pi}=0$, as desired.

From Proposition 3.5 we know that $\left\{T_{n}^{1}(a)\right\}^{\pi}$ and $\left\{T_{n}^{1}(c)\right\}^{\pi}$ commute whenever $a$ is in $P C_{d}$ and $a_{\infty}$ has only finitely many jumps and $c$ belongs to $C(\overline{\mathbb{C}})$. Since the collection of $P C_{d}$ functions $a$ for which $a_{\infty}$ has at most finitely many jumps is dense in $P C_{d}$, it results that $\left\{T_{n}^{1}(a)\right\}^{\pi}$ and $\left\{T_{n}^{1}(c)\right\}^{\pi}$ commute for every $a \in P C_{d}$ and every $c \in C(\overline{\mathbb{C}})$. Consequently, $\mathfrak{C}^{\pi}$ is a $C^{*}$-subalgebra of the center of $\mathfrak{B}^{\pi}$ and so Theorem 2.2 may be applied with $B=\mathfrak{B}^{\pi}$ and $C=\mathfrak{C}^{\pi}$.

From Theorem 2.1 and the fact that $\mathfrak{C}^{\pi} \cong \mathcal{C}^{\pi}$, we know that $M\left(\mathfrak{C}^{\pi}\right)=\mathbb{T}$. For $\tau \in \mathbb{T}$, the maximal ideal of $\mathfrak{C}^{\pi}$ corresponding to $\tau$ is

$$
\left\{\left\{T_{n}^{1}(c)\right\}^{\pi}: c \in C(\overline{\mathbb{C}}), c_{\infty}(\tau)=0\right\},
$$

and the smallest closed two-sided star-ideal $\mathfrak{Y}_{\tau}$ of $\mathfrak{B}^{\pi}$ containing the latter ideal is

$$
\left\{\left\{T_{n}^{1}(a)\right\}^{\pi}: a \in P C_{d}, a_{\infty} \text { is continuous at } \tau, a_{\infty}(\tau)=0\right\} .
$$

Let $\chi$ denote the characteristic function of the half-plane $H_{d(\tau), \tau}^{+}$. For $a \in P C_{d}$, we then have

$$
\left\{T_{n}^{1}(a)\right\}^{\pi}+\mathfrak{Y}_{\tau}=\left\{T_{n}^{1}(a(\tau+0) \chi+a(\tau-0)(1-\chi))\right\}^{\pi}+\mathfrak{Y}_{\tau}
$$

and hence the algebra $\mathfrak{B}^{\pi} / \mathfrak{Y}_{\tau}$ is generated by the identity element and $\left\{T_{n}^{1}(\chi)\right\}^{\pi}+\mathfrak{Y}_{\tau}$. A little thought shows that the spectrum of $\left\{T_{n}^{1}(\chi)\right\}^{\pi}+\mathfrak{Y}_{\tau}$ is contained in $[0,1]$ and that it contains the spectrum of $\left[T^{1}(\chi)\right]^{\pi}+\mathcal{Y}_{\tau}$ in $\mathcal{B}^{\pi} / \mathcal{Y}_{\tau}$. Since the latter spectrum is all of $[0,1]$, we conclude that the spectrum of $\left\{T_{n}^{1}(\chi)\right\}^{\pi}+\mathfrak{Y}_{\tau}$ is also the entire interval $[0,1]$. Consequently, the $C^{*}$-algebra $\mathfrak{B}^{\pi} / \mathfrak{Y}_{\tau}$ is isometrically isomorphic to $C[0,1]$, as is the algebra $\mathcal{B}^{\pi} / \mathcal{Y}_{\tau}$.

From (7) and (19) we see that via the Gelfand transform $\left[T^{1}(a)\right]^{\pi}+\mathcal{Y}_{\tau}$ and $\left\{T_{n}^{1}(a)\right\}^{\pi}+$ $\mathfrak{Y}_{\tau}$ are represented by the same function in $C[0,1]$. Since $\mathcal{B}^{\pi}$ and $\mathfrak{B}^{\pi}$, respectively, are generated by all $\left[T^{1}(a)\right]^{\pi}$ and $\left\{T_{n}^{1}(a)\right\}^{\pi}$ with $a \in P C_{d}$, it follows that for every $\left\{A_{n}\right\}^{\pi} \in$ $\mathfrak{B}^{\pi}$ the elements $\left(S_{1}\left\{A_{n}\right\}\right)^{\pi}+\mathcal{Y}_{\tau}$ and $\left\{A_{n}\right\}^{\pi}+\mathfrak{Y}_{\tau}$ are represented by the same function 
in $C[0,1]$. Now Theorem 2.2 implies that if $\left\{A_{n}\right\}^{\pi}$ is any element of $\mathfrak{B}^{\pi}$, then the spectra of $\left\{A_{n}\right\}^{\pi}$ in $\mathfrak{B}^{\pi}$ and of $\left(S_{1}\left\{A_{n}\right\}\right)^{\pi}$ in $\mathcal{B}^{\pi}$ coincide. Since $\delta_{b}$ is clearly surjective, Lemma 3.8 completes the proof.

Here now is our main result.

Theorem 3.9. Let $\left\{A_{n}\right\}+\mathfrak{N} \in \mathfrak{B}$. Then $\left\{A_{n}\right\}$ is stable if and only if $S_{1}\left\{A_{n}\right\}$ and $S_{2}\left\{A_{n}\right\}$ are invertible. In that case

$$
\limsup _{n \rightarrow \infty}\left\|A_{n}^{-1}\right\|=\max \left\{\left\|\left(S_{1}\left\{A_{n}\right\}\right)^{-1}\right\|,\left\|\left(S_{2}\left\{A_{n}\right\}\right)^{-1}\right\|\right\} .
$$

Pro of. A crucial result by Silbermann (see [24] or [7] or Theorem 7.11 of [8]) says that $\left\{A_{n}\right\}$ is stable, i.e. that $\left\{A_{n}\right\}+\mathfrak{N}$ is invertible in $\mathfrak{F} / \mathfrak{N}$, if and only if $S_{1}\left\{A_{n}\right\}$ and $S_{2}\left\{A_{n}\right\}$ are invertible operators and $\left\{A_{n}\right\}^{\pi}$ is invertible in $\mathfrak{B}^{\pi}$. Proposition 3.7 tells us that $\left\{A_{n}\right\}^{\pi}$ is automatically invertible if only $S_{1}\left\{A_{n}\right\}$ (and thus all the more $\left(S_{1}\left\{A_{n}\right\}\right)^{\pi}$ ) is invertible. This proves the first part of the theorem.

To show (20), denote by $\mathcal{L} \oplus \mathcal{L}$ the $C^{*}$-algebra of all ordered pairs $(A, B)$ of operators $A, B \in \mathcal{L}$ with componentwise algebraic operations and the norm

$$
\|(A, B)\|=\max \{\|A\|,\|B\|\} .
$$

From Proposition 3.6 we know that the mapping

$$
\sigma: \mathfrak{B} \rightarrow \mathcal{L} \oplus \mathcal{L},\left\{A_{n}\right\}+\mathfrak{N} \mapsto\left(S_{1}\left\{A_{n}\right\},\left(S_{2}\left\{A_{n}\right\}\right)\right)
$$

is a well-defined $C^{*}$-algebra homomorphism, and from what was proved in the preceding paragraph, we know that the spectrum of $\left\{A_{n}\right\}+\mathfrak{N}$ in $\mathfrak{F} / \mathfrak{N}$ and thus in the $C^{*}$-subalgebra $\mathfrak{B}$ of $\mathfrak{F} / \mathfrak{N}$ coincides with the spectrum of the element $\sigma\left(\left\{A_{n}\right\}+\mathfrak{N}\right)$ in $\mathcal{L} \oplus \mathcal{L}$. So Lemma 3.8 implies that $\sigma$ is an isometric $C^{*}$-algebra isomorphism of $\mathfrak{B}$ onto $\sigma(\mathfrak{B})$. Hence, if $\left\{A_{n}\right\}+\mathfrak{N}$ is invertible in $\mathfrak{B}$, then

$$
\begin{aligned}
\limsup _{n \rightarrow \infty}\left\|A_{n}^{-1}\right\| & =\left\|\left\{A_{n}^{-1}\right\}+\mathfrak{N}\right\| \\
& =\max \left(\left\|S_{1}\left\{A_{n}^{-1}\right\}\right\|,\left\|S_{2}\left\{A_{n}^{-1}\right\}\right\|\right) \\
& =\max \left(\left\|\left(S_{1}\left\{A_{n}\right\}\right)^{-1}\right\|,\left\|\left(S_{2}\left\{A_{n}\right\}\right)^{-1}\right\|\right) .
\end{aligned}
$$

And here are two first consequences of Theorem 3.9.

THEOREM 3.10. Let $\left\{a_{j k}\right\}$ be a finite collection of functions in $P C_{d}$ and put

$$
A=\sum_{j} \prod_{k} T^{1}\left(a_{j k}\right), \quad A_{n}=\sum_{j} \prod_{k} T_{n}^{1}\left(a_{j k}\right) .
$$

Then the following are equivalent:

(i) the operators $A_{n}$ are invertible in $\operatorname{Im} P_{n}$ for all sufficiently large $n$ and for each $g \in A^{2}(\mathbb{C}, d \mu)$ the unique solution $f_{n} \in \operatorname{Im} P_{n}$ of $A_{n} f_{n}=P_{n} g$ converges in $A^{2}(\mathbb{C}, d \mu)$ to a solution of the equation $A f=g$;

(ii) $A$ and $A^{0}:=\sum_{j} \prod_{k} T^{0}\left(a_{j k}^{0}\right)$ are invertible.

Proof. From Proposition 3.6 we have $S_{1}\left\{A_{n}\right\}=A$ and $S_{2}\left\{A_{n}\right\}=A^{0}$. Assertion (i) is equivalent to the invertibility of $A$ and the stability of $\left\{A_{n}\right\}$ (see, e.g., Proposition 7.3 of [8]), and therefore the equivalence (i) $\Leftrightarrow($ ii) follows immediately from Theorem 3.9. 
Theorem 3.11. Let $a \in P C_{d}$. Then the finite section method is applicable to $T^{1}(a)$ if and only if $T^{1}(a)$ is invertible.

Proof. The "only if" part is a special case of the preceding theorem. If $T^{1}(a)$ is invertible, we deduce from Theorem 2.4 that $0 \in a^{\#}$ and that the winding number of $a^{\#}$ about the origin is zero. This in turn implies that $T^{0}\left(a^{0}\right)$ is invertible. The "if part" is now immediate from the implication (ii) $\Rightarrow(\mathrm{i})$ of the previous theorem.

Two more sets of problems that can be tackled with the help of Theorem 3.9 will be considered in the next two sections.

4. Spectral approximation. The purpose of this section is to show how Theorem 3.9 can be used to gain insight in the relation between the spectrum of $T^{1}(a)$ and the spectrum (eigenvalues) of $T_{n}^{1}(a)$ for large $n$.

Given a sequence $\left\{E_{n}\right\}_{n=0}^{\infty}$ of sets $E_{n} \subset \mathbb{C}$, we denote by $\lim _{n \rightarrow \infty} E_{n}$ the set of all $\lambda \in \mathbb{C}$ with the following property: there are $n_{1}<n_{2}<\ldots$ and $\lambda_{k} \in E_{n_{k}}$ such that $\lambda_{k} \rightarrow \lambda$. The spectrum of an operator $A$ will be denoted by $\Lambda_{0}(A)$, i.e.

$$
\Lambda_{0}(A)=\{\lambda \in \mathbb{C}: A-\lambda I \text { is not invertible }\} .
$$

Theorem 3.11 says that if $T^{1}(a)$ is invertible, then so is $T_{n}^{1}(a)$ for all sufficiently large $n$ and we have

$$
\limsup _{n \rightarrow \infty}\left\|\left[T_{n}^{1}(a)\right]^{-1}\right\|<\infty .
$$

This is all we need to establish the inclusion

$$
\lim _{n \rightarrow \infty} \Lambda_{0}\left(T_{n}^{1}(a)\right) \subset \Lambda_{0}\left(T^{1}(a)\right) .
$$

Indeed, if $\lambda \notin \Lambda_{0}\left(T^{1}(a)\right)$, then there are an $n_{0}$ and an $M$ such that

$$
\left\|\left[T_{n}^{1}(a-\lambda)\right]^{-1}\right\| \leq M \quad \text { for all } n \geq n_{0},
$$

and since

$$
\left\|\left[T_{n}^{1}(a-\mu)\right]^{-1}\right\| \leq \frac{\left\|\left[T_{n}^{1}(a-\lambda)\right]^{-1}\right\|}{1-|\mu-\lambda|\left\|\left[T_{n}^{1}(a-\lambda)\right]^{-1}\right\|}
$$

whenever $|\mu-\lambda|<1 / M$, it follows that

$$
\left\|\left[T_{n}^{1}(a-\mu)\right]^{-1}\right\| \leq 2 M
$$

if $n \geq n_{0}$ and $\mu \in U_{1 /(2 M)}(\lambda)=\{\zeta \in \mathbb{C}:|\zeta-\lambda|<1 /(2 M)\}$. Hence,

$$
U_{1 /(2 M)}(\lambda) \cap \Lambda_{0}\left(T_{n}^{1}(a)\right)=\emptyset
$$

for $n \geq n_{0}$ and thus $\lambda \notin \lim _{n \rightarrow \infty} \Lambda_{0}\left(T_{n}^{1}(a)\right)$.

From the work of Schmidt and Spitzer [22] we know that the limit set $\lim _{n \rightarrow \infty}$ $\Lambda_{0}\left(T_{n}^{0}(a)\right)$ may be much smaller than $\Lambda_{0}\left(T^{0}(a)\right)$, and therefore we conjecture that in general the inclusion in (22) is also "strict in some sense". Instead of pursuing this question, we will show how Theorem 3.9 produces fairly sharp results for so-called pseudospectra.

Pseudospectra were considered by Reichel and Trefethen [21]. For $\varepsilon>0$, the $\varepsilon$ pseudospectrum $\Lambda_{\varepsilon}(A)$ of an operator $A$ is defined as

$$
\Lambda_{\varepsilon}(A)=\left\{\lambda \in \mathbb{C}: \lambda \in \Lambda_{0}(A) \text { or }\left\|(A-\lambda I)^{-1}\right\| \geq 1 / \varepsilon\right\} .
$$


Using a result of Widom [27], Reichel and Trefethen [21] have shown that

$$
\lim _{n \rightarrow \infty} \Lambda_{\varepsilon}\left(T_{n}^{0}(a)\right)=\Lambda_{\varepsilon}\left(T^{0}(a)\right) .
$$

We here prove the Segal-Bargmann space analogue of this by having recourse to Theorem 3.9 and the approach of $[6]$.

Estimating the limit set of the pseudospectra of operator sequences from below leads to the problem whether the resolvent of an operator can have constant norm. We conjectured that this cannot happen but had not been able to prove this. Therefore we posed the problem at the Banach Semester in Warsaw in March, 1994. Only a few weeks later, Andrzej Daniluk of Cracow sent us the following e-mail:

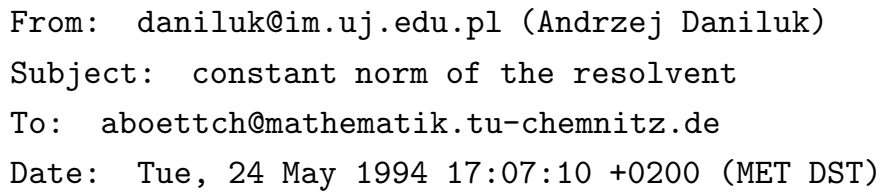

Dear Sir, Visiting the Banach center in March you asked the following question:

Can the norm of the resolvent $R(z, T)$ be constant for every $z$ in the unit disk? Unfortunately $I$ don't remember if you considered $T$ being an element of any Banach algebra or an operator on a Hilbert (Banach?) space. As you probably know, I found the NEGATIVE answer to your question in the second case but I didn't in general. I'm sorry that I'm writing you so late but I got your e-mail address only just yesterday.

So now I'm going to sketch you the proof. The same result can however be obtained when $T$ acts not on a Hilbert space but on a Banach space with a positive modulus of convexity (so in particular on $L^{p}$ ). Although the reasoning is the same in both cases, the proof is nicer for Hilbert spaces and I'll concentrate on it.

Let us assume that the norm $|R(z, T)|=M$, for $|z| \leq 1$. As

$$
R(z, T)=\sum_{j} z^{j} T^{-j-1}
$$

we easily compute for any vector $f$

$$
|R(z, T) f|^{2}=\sum_{j, k} z^{j} z^{-k}\left\langle T^{-j-1} f, T^{-k-1} f\right\rangle
$$

and its integral mean

$$
\frac{1}{2 i \pi} \int_{|z|=1}|R(z, T) f|^{2} d z=\sum_{j}\left|T^{-j-1} f\right|^{2},
$$

which is by assumption less than or equal to $M^{2}|f|^{2}$. But as $\left|T^{-1}\right|=M$ we can find a normalized vector $f$ such that $\left|T^{-1} f\right|^{2}>M^{2}-\varepsilon$. So every other component in the last sum above must be less than $\varepsilon$. Particularly, 0 must be then in the approximate spectrum of $T^{-2}$, which is a contradiction.

Yours faithfully, Andrzej Daniluk 
Thus, Andrzej Daniluk solved the problem we could not solve, and both the following result and its proof are due to him $\left(^{1}\right)$.

Proposition 4.1. Let $H$ be a Hilbert space and let $T \in \mathcal{L}(H)$. Suppose $T-\lambda I$ is invertible for all $\lambda$ in some open subset $U$ of $\mathbb{C}$ and $\left\|(T-\lambda I)^{-1}\right\| \leq M$ for all $\lambda \in U$. Then $\left\|(T-\lambda I)^{-1}\right\|<M$ for all $\lambda \in U$.

Proof. A little thought reveals that what we must show is the following: if $U$ is an open subset of $\mathbb{C}$ containing the origin and $\left\|(T-\lambda I)^{-1}\right\| \leq M$ for all $\lambda \in U$, then $\left\|T^{-1}\right\|<M$. To prove this, assume the contrary, i.e. let $\left\|T^{-1}\right\|=M$. We have

$$
(T-\lambda I)^{-1}=\sum_{j=0}^{\infty} \lambda^{j} T^{-j-1}
$$

for all $\lambda$ in some disk $|\lambda| \leq r$. Given any $f \in H$, we therefore get

$$
\left\|(T-\lambda I)^{-1} f\right\|^{2}=\sum_{j, k \geq 0} \lambda^{j} \bar{\lambda}^{k}\left(T^{-j-1} f, T^{-k-1} f\right)
$$

whenever $|\lambda| \leq r$. Integrating the latter equality along the circle $|\lambda|=r$ we obtain

$$
\frac{1}{2 \pi} \int_{|\lambda|=r}\left\|(T-\lambda I)^{-1} f\right\|^{2}|d z|=\sum_{j=0}^{\infty} r^{2 j}\left\|T^{-j-1} f\right\|^{2},
$$

and since $\left\|(T-\lambda I)^{-1} f\right\| \leq M\|f\|$, we arrive at the inequality

$$
\left\|T^{-1} f\right\|^{2}+r^{2}\left\|T^{-2} f\right\|^{2} \leq \sum_{j=0}^{\infty} r^{2 j}\left\|T^{-j-1} f\right\|^{2} \leq M^{2}\|f\|^{2} .
$$

Now pick an arbitrary $\varepsilon>0$. Because $\left\|T^{-1}\right\|=M$ by assumption, there is an $f_{\varepsilon} \in H$ such that $\left\|f_{\varepsilon}\right\|=1$ and $\left\|T^{-1} f_{\varepsilon}\right\|^{2}>M^{2}-\varepsilon$. It follows that

$$
M^{2}-\varepsilon+r^{2}\left\|T^{-2} f_{\varepsilon}\right\|^{2}<M^{2},
$$

i.e. $\left\|T^{-2} f_{\varepsilon}\right\|^{2}<\varepsilon r^{-2}$, and consequently,

$$
1=\left\|f_{\varepsilon}\right\|^{2} \leq\left\|T^{2}\right\|^{2}\left\|T^{-2} f_{\varepsilon}\right\|^{2}<\varepsilon r^{-2}\left\|T^{2}\right\|^{2},
$$

which is impossible if $\varepsilon>0$ is sufficiently small. This contradiction shows that $\left\|T^{-1}\right\|$ $<M$.

Now we are in a position to give a lower bound for the limit set of the pseudospectra of certain sequences of operators.

Proposition 4.2. Let $H$ be a Hilbert space, and let $P_{n} \in \mathcal{L}(H)$ be a sequence of projections converging strongly to the identity operator. If $A \in \mathcal{L}(H)$ is any operator and $A_{n} \in \mathcal{L}\left(\operatorname{Im} P_{n}\right)$ any sequence of operators such that $A_{n} \rightarrow A$ and $A_{n}^{*} \rightarrow A^{*}$ strongly, then

$$
\Lambda_{\varepsilon}(A) \subset \lim _{n \rightarrow \infty} \Lambda_{\varepsilon}\left(A_{n}\right)
$$

for each $\varepsilon>0$.

$\left({ }^{1}\right)$ Editorial note: See also J. Globevnik, Schwarz's lemma for the spectral radius, Rev. Roumaine Math. Pures Appl. 19 (1974), 1009-1012. 
Proof. Let first $\lambda \in \Lambda_{0}(A)$. We claim that then

$$
\limsup _{n \rightarrow \infty}\left\|\left(A_{n}-\lambda I\right)^{-1}\right\|=\infty \text {. }
$$

Indeed, if $\left\|\left(A_{n}-\lambda I\right)^{-1}\right\| \leq M$ for $n \geq n_{0}$, then $\left\|P_{n} \varphi\right\| \leq M\left\|\left(A_{n}-\lambda I\right) P_{n} \varphi\right\|$ for all $\varphi \in H$, and passage to limit $n \rightarrow \infty$ gives $\|\varphi\| \leq M\|(A-\lambda I) \varphi\|$ for all $\varphi \in H$, implying that the range of $A-\lambda I$ is closed and that $A-\lambda I$ is injective. Considering adjoints we similarly get $\|\varphi\| \leq M\left\|\left(A^{*}-\bar{\lambda} I\right) \varphi\right\|$ for all $\varphi \in H$, which shows that the range of $A-\lambda I$ is dense. Hence, if (24) is not valid then $A-\lambda I$ is invertible. Our assumption that $\lambda$ be in $\Lambda_{0}(A)$ therefore implies that (24) holds. From (24) we infer that there is a sequence $n_{k} \rightarrow \infty$ such that $\lambda \in \Lambda_{\varepsilon}\left(A_{n_{k}}\right)$ and thus, $\lambda \in \lim _{n \rightarrow \infty} \Lambda_{\varepsilon}\left(A_{n}\right)$.

Now suppose $\lambda \in \Lambda_{\varepsilon}(A) \backslash \Lambda_{0}(A)$. Then $A-\lambda I$ is invertible and $\left\|(A-\lambda I)^{-1}\right\| \geq 1 / \varepsilon$. Let $U$ be any open neighborhood of $\lambda$. From Proposition 4.1 we deduce that there is a $\mu \in U$ such that $\left\|(A-\mu I)^{-1}\right\|>1 / \varepsilon$ and thus $\left\|(A-\mu I)^{-1}\right\|>1 /(\varepsilon-1 / k)$ for all sufficiently large $k$. Consequently, we have $\lambda=\lim _{k \rightarrow \infty} \lambda_{k}$ with $\lambda_{k} \in \Lambda_{1 / \varepsilon-1 / k}(A)$. If $T$ is any invertible Hilbert space operator, then

$$
\left\|T^{-1}\right\|=\sup _{\psi \neq 0} \frac{\left\|T^{-1} \psi\right\|}{\|\psi\|}=\sup _{\varphi \neq 0} \frac{\|\varphi\|}{\|T \varphi\|}=\left(\inf _{\varphi=0} \frac{\|T \varphi\|}{\|\varphi\|}\right)^{-1}
$$

Hence, for each $n$, there exists a $\varphi$ in $H$ such that

$$
\|\varphi\|=1, \quad\left\|\left(A-\lambda_{k} I\right) \varphi\right\|<\varepsilon-1 /(2 k) .
$$

We have

$$
\begin{aligned}
\left\|\left(A_{n}-\lambda_{k} I\right) P_{n} \varphi\right\| & \leq\left\|\left(A_{n}-A\right) P_{n} \varphi\right\|+\left\|\left(A-\lambda_{k} I\right) P_{n} \varphi\right\| \\
& \leq\left\|\left(A_{n}-A\right) P_{n} \varphi\right\|+\left\|\left(A-\lambda_{k} I\right) \varphi\right\|+\left\|\left(A-\lambda_{k} I\right)\left(I-P_{n}\right) \varphi\right\|
\end{aligned}
$$

and since $\left(A_{n}-A\right) P_{n}$ and $I-P_{n}$ converge strongly to zero, we obtain

$$
\left\|\left(A_{n}-\lambda_{k} I\right) P_{n} \varphi\right\|<\varepsilon-1 /(3 k)
$$

for all sufficiently large $n$. Because $\left\|P_{n} \varphi\right\|$ tends to $\|\varphi\|=1$ as $n \rightarrow \infty$, we get

$$
\left\|\left(A_{n}-\lambda_{k} I\right) P_{n} \varphi\right\| /\left\|P_{n} \varphi\right\|<\varepsilon-1 /(4 k)<\varepsilon
$$

for all $n$ large enough. Now (25) implies that

$$
\left\|\left(A_{n}-\lambda_{k} I\right)^{-1}\right\|>1 / \varepsilon
$$

and consequently, $\lambda_{k} \in \Lambda_{\varepsilon}\left(A_{n}\right)$ for all sufficiently large $n$. This proves that $\lambda=\lim _{k \rightarrow \infty} \lambda_{k}$ belongs to $\lim _{n \rightarrow \infty} \Lambda_{\varepsilon}\left(A_{n}\right)$.

The following result sharpens (21).

Theorem 4.3. Let $a \in P C_{d}$ and suppose (21) holds. Then $T^{1}(a)$ and $T^{0}\left(a^{0}\right)$ are invertible, the limit $\lim _{n \rightarrow \infty}\left\|\left[T_{n}^{1}(a)\right]^{-1}\right\|$ exists, and

$$
\lim _{n \rightarrow \infty}\left\|\left[T_{n}^{1}(a)\right]^{-1}\right\|=\max \left\{\left\|\left[T^{1}(a)\right]^{-1}\right\|,\left\|\left[T^{0}\left(a^{0}\right)\right]^{-1}\right\|\right\} .
$$

Proof. Theorem 3.9 with $A_{n}=T_{n}^{1}(a)$ implies the invertibility of $T^{1}(a)$ and $T^{0}\left(a^{0}\right)$ and yields (26) with limsup in place of lim. Since $\left[T_{n}^{1}(a)\right]^{-1}$ converges strongly to $\left[T^{1}(a)\right]^{-1}$, it follows that

$$
\liminf _{n \rightarrow \infty}\left\|\left[T_{n}^{1}(a)\right]^{-1}\right\| \geq\left\|\left[T^{1}(a)\right]^{-1}\right\|,
$$


and because $\left[T^{0}\left(a^{0}\right)\right]^{-1}$ is the strong limit of $W_{n}\left[T_{n}^{1}(a)\right]^{-1} W_{n}$, we obtain

$$
\liminf _{n \rightarrow \infty}\left\|\left[T_{n}^{1}(a)\right]^{-1}\right\| \geq \liminf _{n \rightarrow \infty}\left\|W_{n}\left[T_{n}^{1}(a)\right]^{-1} W_{n}\right\|=\left\|\left[T^{0}\left(a^{0}\right)\right]^{-1}\right\| .
$$

TheOREM 4.4. Let $a \in P C_{d}$. Then for each $\varepsilon>0$,

$$
\lim _{n \rightarrow \infty} \Lambda_{\varepsilon}\left(T_{n}^{1}(a)\right)=\Lambda_{\varepsilon}\left(T^{1}(a)\right) \cup \Lambda_{\varepsilon}\left(T^{0}\left(a^{0}\right)\right) .
$$

Furthermore,

$$
\lim _{\varepsilon \rightarrow 0} \lim _{n \rightarrow \infty} \Lambda_{\varepsilon}\left(T_{n}^{1}(a)\right)=\Lambda_{0}\left(T^{1}(a)\right) .
$$

Proof. Proposition 4.2 with $A_{n}=T_{n}^{1}(a)$ shows that $\Lambda_{\varepsilon}\left(T^{1}(a)\right)$ is contained in $\lim _{n \rightarrow \infty} \Lambda_{\varepsilon}\left(T_{n}^{1}(a)\right)$. Because

$$
\left\|\left[W_{n} T_{n}^{1}(a-\lambda) W_{n}\right]^{-1}\right\|=\left\|W_{n}\left[T_{n}^{1}(a-\lambda)\right]^{-1} W_{n}\right\| \leq\left\|\left[T_{n}^{1}(a-\lambda)\right]^{-1}\right\|,
$$

we have

$$
\Lambda_{\varepsilon}\left(W_{n} T_{n}^{1}(a) W_{n}\right) \subset \Lambda_{\varepsilon}\left(T_{n}^{1}(a)\right),
$$

and hence Proposition 4.2 with $A_{n}=W_{n} T_{n}^{1}(a) W_{n}$ and $A_{n}^{*}=W_{n} T_{n}^{1}(\bar{a}) W_{n}$ implies that $\Lambda_{\varepsilon}\left(T^{0}\left(a^{0}\right)\right)$ is a subset of $\lim _{n \rightarrow \infty} \Lambda_{\varepsilon}\left(T_{n}^{1}(a)\right)$.

To prove the reverse inclusion of $(27)$, assume

$$
\lambda \notin \Lambda_{\varepsilon}\left(T^{1}(a)\right) \cup \Lambda_{\varepsilon}\left(T^{0}\left(a^{0}\right)\right) .
$$

Then $\left\|\left[T^{1}(a-\lambda)\right]^{-1}\right\|<1 / \varepsilon$ and $\left\|\left[T^{0}\left(a^{0}-\lambda\right)\right]^{-1}\right\|<1 / \varepsilon$, and from (26) we conclude that

$$
\left\|\left[T_{n}^{1}(a-\lambda)\right]^{-1}\right\| \leq 1 / \varepsilon-\delta<1 / \varepsilon
$$

for all $n \geq n_{0}$. Taking into account the estimate (23), we see that if $\mu$ is in a sufficiently small neighborhood $U_{\eta}(\lambda)$ of $\lambda$, then

$$
\left\|\left[T_{n}^{1}(a-\mu)\right]^{-1}\right\|<1 / \varepsilon-\delta / 2<1 / \varepsilon
$$

for all $n \geq n_{0}$. Consequently, $U_{\eta}(\lambda) \cap \Lambda_{\varepsilon}\left(T_{n}^{1}(a)\right)=\emptyset$ for all $n \geq n_{0}$, implying that $\lambda \notin \lim \Lambda_{\varepsilon}\left(T_{n}^{1}(a)\right)$.

Finally, since

$$
\lim _{\varepsilon \rightarrow 0} \Lambda_{\varepsilon}(A)=\Lambda_{0}(A)
$$

for every operator $A$, equality (27) gives

$$
\lim _{\varepsilon \rightarrow 0} \lim _{n \rightarrow \infty} \Lambda_{\varepsilon}\left(T_{n}^{1}(a)\right)=\Lambda_{0}\left(T^{1}(a)\right) \cup \Lambda_{0}\left(T^{0}\left(a^{0}\right)\right)
$$

and Theorem 2.4 in conjunction with the well-known invertibility criterion for Hardy space Toeplitz operators with piecewise continuous symbols (see [17]) shows that $\Lambda_{0}\left(T^{0}\left(a^{0}\right)\right) \subset \Lambda_{0}\left(T^{1}(a)\right)$

We remark that (26) and (27) can be extended to the situation considered in Theorem 3.10. Namely, define $A_{n}, A$, and $A^{0}$ as in that theorem. Then if $\left\{A_{n}\right\}$ is a stable sequence, the operators $A$ and $A^{0}$ are invertible, the limit $\lim _{n \rightarrow \infty}\left\|A_{n}^{-1}\right\|$ exists, and

$$
\lim _{n \rightarrow \infty}\left\|A_{n}^{-1}\right\|=\max \left\{\left\|A^{-1}\right\|,\left\|\left[A^{0}\right]^{-1}\right\|\right\} .
$$

Moreover, if $\varepsilon>0$, then

$$
\lim _{n \rightarrow \infty} \Lambda_{\varepsilon}\left(A_{n}\right)=\Lambda_{\varepsilon}(A) \cup \Lambda_{\varepsilon}\left(A^{0}\right) .
$$

The proofs are the same as those of Theorems 4.3 and 4.4 . 
5. Collocation methods. A collocation method for approximately solving equation (2) consists in the following. Choose $n+1$ points $z_{0}, z_{1}, \ldots, z_{n} \in \mathbb{C}$ (the collocation points) and $n+1$ functions $\varphi_{0}, \varphi_{1}, \ldots, \varphi_{n} \in A^{2}(\mathbb{C}, d \mu)$ (the basis functions). Look for an approximate solution $f_{n}$ in the form of a linear combination

$$
f_{n}(w)=c_{0} \varphi_{0}(w)+c_{1} \varphi_{1}(w)+\ldots+c_{n} \varphi_{n}(w) \quad(w \in \mathbb{C})
$$

and determine the $n+1$ unknown coefficients so that $\left(T^{1}(a) f_{n}\right)(z)$ and $g(z)$ coincide at the $n+1$ points $z=z_{0}, z_{1}, \ldots, z_{n}$. Notice that the $n+1$ equations $\left(T^{1}(a) f_{n}\right)\left(z_{k}\right)=g\left(z_{k}\right)$ are the linear algebraic system

$$
\sum_{j=0}^{n} c_{j} \int_{\mathbb{C}} a(w) e^{z_{k} \bar{w} / 2} \varphi_{j}(w) d \mu(w)=g\left(z_{k}\right) \quad(k=0,1, \ldots, n) .
$$

When studying the problem of the convergence of a collocation method, one has to choose for each $n$ collocation points $z_{0}^{(n)}, z_{1}^{(n)}, \ldots, z_{n}^{(n)}$ and basis functions $\varphi_{0}^{(n)}, \varphi_{1}^{(n)}, \ldots, \varphi_{n}^{(n)}$.

We here choose the following system of collocation points: fix $r>0$, and let $z_{0}^{(n)}, z_{1}^{(n)}, \ldots, z_{n}^{(n)}$ be the zeros of the equation $z^{n+1}-r^{n+1}=0$. Throughout what follows $z_{0}^{(n)}, z_{1}^{(n)}, \ldots, z_{n}^{(n)}$ are always specified in this way.

We say that the collocation method with a system of basis functions $\left\{\varphi_{0}^{(n)}\right.$, $\left.\varphi_{1}^{(n)}, \ldots, \varphi_{n}^{(n)}\right\}(n=0,1,2, \ldots)$ is applicable to $T^{1}(a)$ if there is an $n_{0}$ such that the equations

$$
\sum_{j=0}^{n} c_{j}^{(n)} \int_{\mathbb{C}} a(w) e^{z_{k}^{(n)}} \bar{w} / 2 \varphi_{j}^{(n)}(w) d \mu(w)=g\left(z_{k}^{(n)}\right) \quad(k=0,1, \ldots, n)
$$

are uniquely solvable for all $n \geq n_{0}$ and all $g \in A^{2}(\mathbb{C}, d \mu)$ and the functions $f_{n}$ given by

$$
f_{n}(w)=c_{0}^{(n)} \varphi_{0}^{(n)}(w)+c_{1}^{(n)} \varphi_{1}^{(n)}(w)+\ldots+c_{n}^{(n)} \varphi_{n}^{(n)}(w) \quad(w \in \mathbb{C})
$$

converge in $A^{2}(\mathbb{C}, d \mu)$ to a solution $f \in A^{2}(\mathbb{C}, d \mu)$ of the original equation $T^{1}(a) f=g$.

In case we choose $\left\{\varphi_{0}^{(n)}, \ldots, \varphi_{n}^{(n)}\right\}=\left\{e_{0}, \ldots, e_{n}\right\}$ as the basis functions, where the monomials $e_{j}$ are given by (4), we speak of polynomial collocation.

Theorem 5.1. Let $a \in P C_{d}$. Then polynomial collocation is applicable to $T^{1}(a)$ if and only if $T^{1}(a)$ is invertible.

Another choice of the basis functions is

$$
\left\{\varphi_{0}^{(n)}, \ldots, \varphi_{n}^{(n)}\right\}=\left\{K_{z_{0}^{(n)}}, \ldots, K_{z_{n}^{(n)}}\right\}
$$

where, for $z \in \mathbb{C}$, the function $K_{z} \in A^{2}(\mathbb{C}, d \mu)$ is given by

$$
K_{z}(w)=e^{\bar{z} w / 2}(w \in \mathbb{C}) .
$$

If the basis functions are chosen in this way, we speak of analytic element collocation.

Theorem 5.2. Let $a \in P C_{d}$. Then analytic element collocation is applicable to $T^{1}(a)$ if and only if $T^{1}(a)$ is invertible.

The Bergman space analogues of the above two theorems were established in [11]. Therefore we here confine us to merely pointing out the main ideas of their proofs.

Recall that $P_{n}$ is the orthogonal projection of $A^{2}(\mathbb{C}, d \mu)$ onto the space $\operatorname{Im} P_{n}$ of all polynomials of degree at most $n$. We denote by $L_{n}: A^{2}(\mathbb{C}, d \mu) \rightarrow \operatorname{Im} P_{n}$ the Lagrange 
interpolation projection, that is, the projection that sends $f \in A^{2}(\mathbb{C}, d \mu)$ to the (unique) polynomial $L_{n} f \in \operatorname{Im} P_{n}$ satisfying $\left(L_{n} f\right)\left(z_{k}^{(n)}\right)=f\left(z_{k}^{(n)}\right)$ for $k=0, \ldots, n$. If we put

$$
q_{j}^{(n)}(z)=\prod_{k \neq j} \frac{z-z_{j}}{z_{k}-z_{j}} \quad(z \in \mathbb{C}),
$$

then $q_{j}^{(n)} \in \operatorname{Im} P_{n}$ and we have

$$
L_{n} f=\sum_{j=0}^{n} f\left(z_{j}^{(n)}\right) q_{j}^{(n)}
$$

Now the functions $K_{z}(w)=e^{\bar{z} w / 2}$ enter the scene. Equality (1) just says that

$$
f(z)=\left(f, K_{z}\right) \text { for all } f \in A^{2}(\mathbb{C}, d \mu) \text { and all } z \in \mathbb{C} .
$$

Hence, we may write (31) in the form

$$
L_{n} f=\sum_{j=0}^{n}\left(f, K_{z_{j}^{(n)}}\right) q_{j}^{(n)},
$$

which immediately implies that the adjoint $L_{n}^{*}$ of $L_{n}$ is the projection of $A^{2}(\mathbb{C}, d \mu)$ onto the linear hull of the functions (30) acting by the rule

$$
L_{n}^{*} f=\sum_{j=0}^{n}\left(f, q_{j}^{(n)}\right) K_{z_{j}^{(n)}} .
$$

In the case of polynomial and analytic element collocation, the equations (29) are equivalent to the equations

$$
L_{n} T^{1}(a) f_{n}=L_{n} g \quad\left(f_{n} \in \operatorname{Im} P_{n}\right)
$$

and

$$
L_{n} T^{1}(a) f_{n}=L_{n} g \quad\left(f_{n} \in \operatorname{Im} L_{n}^{*}\right),
$$

respectively. As in [11] one can show (and this is the crucial point) that

$$
\left\|L_{n}-P_{n}\right\|=\left\|L_{n}^{*}-P_{n}\right\| \rightarrow 0 \text { as } n \rightarrow \infty .
$$

Since $P_{n} T^{1}(a) P_{n}: \operatorname{Im} P_{n} \rightarrow \operatorname{Im} P_{n}$ is stable if and only if $T^{1}(a)$ is invertible (Theorem 3.11 ), it follows easily from (32) that the stability of sequences given by

$$
L_{n} T^{1}(a) P_{n}: \operatorname{Im} P_{n} \rightarrow \operatorname{Im} P_{n}, L_{n} T^{1}(a) L_{n}^{*}: \operatorname{Im} L_{n}^{*} \rightarrow \operatorname{Im} P_{n}
$$

is equivalent to the invertibility of $T^{1}(a)$. This proves Theorems 5.1 and 5.2.

6. Higher dimensions. The Segal-Bargmann space $A^{2}\left(\mathbb{C}^{N}, d \mu\right)$ is the Hilbert space of all functions $f$ which are analytic on $\mathbb{C}^{N}$ and satisfy

$$
\|f\|^{2}:=\int_{\mathbb{C}^{N}}\left|f\left(z_{1}, \ldots, z_{N}\right)\right|^{2} d \mu\left(z_{1}\right) \ldots d \mu\left(z_{N}\right)<\infty .
$$

Since the space $A^{2}\left(\mathbb{C}^{N}, d \mu\right)$ is the Hilbert space tensor product of $N$ copies of $A^{2}(\mathbb{C}, d \mu)$, many of the results established in the preceding sections can be carried over to $A^{2}\left(\mathbb{C}^{N}, d \mu\right)$ by means of the techniques developed in [7] and [10]. We therefore limit ourselves to only formulating the results. Moreover, in order to avoid unnecessary notational complications, we will mainly consider the case $N=2$. 
The Toeplitz operator on $A^{2}\left(\mathbb{C}^{2}, d \mu\right)$ induced by a function $a \in L^{\infty}\left(\mathbb{C}^{2}\right)$ will be denoted by $T^{1,1}(a)$. It is defined by

$$
\left(T^{1,1}(a) f\right)(z)=\int_{\mathbb{C}^{2}} a(w) e^{z \bar{w} / 2} f(w) d \mu(w) \quad\left(z \in \mathbb{C}^{2}\right)
$$

with $z \bar{w}=z_{1} \overline{w_{1}}+z_{2} \overline{w_{2}}$ and $d \mu(w)=d \mu\left(w_{1}\right) d \mu\left(w_{2}\right)$. Let $P C_{d} \odot P C_{d}$ denote the algebraic tensor product of two copies of $P C_{d}$, i.e. the collection of all functions $a$ on $\mathbb{C}^{2}$ which are representable as a finite sum of the form

$$
a\left(z_{1}, z_{2}\right)=\sum_{j} b_{j}\left(z_{1}\right) c_{j}\left(z_{2}\right) \quad\left(b_{j}, c_{j} \in P C_{d}\right),
$$

and we let $P C_{d} \otimes P C_{d}$ stand for the closure of $P C_{d} \odot P C_{d}$ in $L^{\infty}\left(\mathbb{C}^{2}\right)$. Notice that if $a$ is of the form (33), then

$$
T^{1,1}(a)=\sum_{j} T^{1}\left(b_{j}\right) \otimes T^{1}\left(c_{j}\right)
$$

The role played by the operator $T^{0}(a)$ in the foregoing sections is now taken upon by three operators $T^{1,0}(a), T^{0,1}(a), T^{0,0}(a)$. In case $a$ is given by (33), we put

$$
T^{\gamma, \delta}(a)=\sum_{j} T^{\gamma}\left(b_{j}\right) \otimes T^{\delta}\left(c_{j}\right)
$$

for $\gamma, \delta \in\{0,1\}$. For functions $a \in P C_{d} \otimes P C_{d}$, the "mixed" operators $T^{\varepsilon, \delta}(a)$ may be defined via approximation of $a$ by symbols $a_{n} \in P C_{d} \odot P C_{d}$. Here is an alternative way of defining $T^{\gamma, \delta}(a)$ for general $a \in P C_{d} \otimes P C_{d}$. We know from Proposition 3.6 that if $b \in P C_{d}$, then $T^{0}(b)$ is the strong limit of $W_{n} T^{1}\left(b^{0}\right) W_{n}$ where $b^{0}(z)=b(\bar{z})$. Hence, for $a \in P C_{d} \otimes P C_{d}$ we might put

$$
a^{1,0}\left(z_{1}, z_{2}\right)=a\left(z_{1}, \overline{z_{2}}\right), a^{0,1}\left(z_{1}, z_{2}\right)=a\left(\overline{z_{1}}, z_{2}\right), a^{0,0}\left(z_{1}, z_{2}\right)=a\left(\overline{z_{1}}, \overline{z_{2}}\right),
$$

and then

$$
\begin{aligned}
& T^{1,0}(a)=\underset{n \rightarrow \infty}{\mathrm{s}-\lim _{n}}\left(P_{n} \otimes W_{n}\right) T^{1,1}\left(a^{1,0}\right)\left(P_{n} \otimes W_{n}\right) \\
& T^{0,1}(a)=\underset{n \rightarrow \infty}{\mathrm{s}-\lim }\left(W_{n} \otimes P_{n}\right) T^{1,1}\left(a^{0,1}\right)\left(W_{n} \otimes P_{n}\right) \\
& T^{0,0}(a)=\underset{n \rightarrow \infty}{\mathrm{s}-\lim _{n}}\left(W_{n} \otimes W_{n}\right) T^{1,1}\left(a^{0,0}\right)\left(W_{n} \otimes W_{n}\right) .
\end{aligned}
$$

One can indeed show that the three strong limits exist and define operators in the $C^{*}$ algebra tensor product $\mathcal{B} \otimes \mathcal{B}$, with $\mathcal{B}$ as in Section 2 . For functions $a \in P C_{d} \odot P C_{d}$, the three operators defined in this way coincide with the operators given by (34).

The orthogonal projection of $A^{2}\left(\mathbb{C}^{2}, d \mu\right)$ onto the linear hull of the monomials $z_{1}^{j_{1}} z_{2}^{j_{2}}$ $\left(j_{1}, j_{2}=0, \ldots, n\right)$ is $P_{n} \otimes P_{n}$. We say that the finite section method is applicable to an operator $A \in \mathcal{L}\left(A^{2}\left(\mathbb{C}^{2}, d \mu\right)\right)$ if there is an $n_{0}$ such that the equations

$$
\left(P_{n} \otimes P_{n}\right) A f_{n}=\left(P_{n} \otimes P_{n}\right) g \quad\left(f_{n} \in \operatorname{Im}\left(P_{n} \otimes P_{n}\right)\right)
$$

are uniquely solvable for all $n \geq n_{0}$ and all $g \in A^{2}\left(\mathbb{C}^{2}, d \mu\right)$ and $f_{n}$ converges in $A^{2}\left(\mathbb{C}^{2}, d \mu\right)$ to a solution $f \in A^{2}\left(\mathbb{C}^{2}, d \mu\right)$ of the equation $A f=g$. 
TheOREm 6.1. Let $a \in P C_{d} \otimes P C_{d}$. Then the finite section method is applicable to $T^{1,1}(a)$ if and only if the four operators

$$
T^{1,1}(a), T^{1,0}\left(a^{1,0}\right), T^{0,1}\left(a^{0,1}\right), T^{0,0}\left(a^{0,0}\right)
$$

are invertible.

Here is the extension of this theorem to mixed Toeplitz operators.

Theorem 6.2. Let $a \in P C_{d} \otimes P C_{d}$ and $(\gamma, \delta) \in\{0,1\}^{2}$. Then the finite section method is applicable to $T^{\gamma, \delta}(a)$ if and only if the four operators

$$
T^{\gamma, \delta}(a), T^{\gamma, 0}\left(a^{1,0}\right), T^{0, \delta}\left(a^{0,1}\right), T^{0,0}\left(a^{0,0}\right)
$$

are invertible.

Of course, Theorem 6.1 motivates the consideration of mixed Toeplitz operators. But what is the motivation for establishing Theorem 6.2? In this connection we first remark that the operator $T^{0,0}(a)$ is unitarily equivalent to a Toeplitz (or discrete Wiener-Hopf) operator on the quarter-plane $\mathbb{Z}_{+} \times \mathbb{Z}_{+}$; thus, Theorem 6.2 comprises the result (proved by Kozak [19] for continuous and in [7] for piecewise continuous symbols) that the finite section method is applicable to $T^{0,0}(a)$ if and only if the four operators

$$
T^{0,0}(a), T^{0,0}\left(a^{1,0}\right), T^{0,0}\left(a^{0,1}\right), T^{0,0}\left(a^{0,0}\right)
$$

are invertible. However, the actual justification of Theorem 6.2 is as follows.

The reader will certainly see how to generalize Theorems 6.1 and 6.2 to dimensions $N \geq 3$ : the four operators occurring in these theorems have then to be replaced by $2^{N}$ operators constructed in an obvious way. We can indeed prove such a generalization, and our proof uses induction on the dimension $N$. However, when proving results by induction, one is occasionally confronted with the phenomenon that such a proof works only if the result to be proved is sufficiently strong. And this is exactly what happens in our situation: it is difficult to extend Theorem 6.1 by induction to higher dimensions, but it is relatively easy to generalize the stronger Theorem 6.2 by induction on $N$ to dimensions $N \geq 2$ (see also [12], p. 30).

Due to what was said in the last two paragraphs, we formulate the following results for mixed Toeplitz operators (although our primary interest is in the "pure" Segal-Bargmann space operators $\left.T^{1,1}(a)\right)$.

Let $a \in P C_{d} \otimes P C_{d}$ and $w \in \mathbb{C}$. Then the functions $\varphi$ and $\psi$ given by $\varphi(z)=a(z, w)$ and $\psi(z)=a(w, z)$ belong to $P C_{d}$ and hence, $\varphi(\tau \pm 0)$ and $\psi(\tau \pm 0)$ are well defined for each $\tau \in \mathbb{T}$. We put

$$
a(\tau \pm 0, w)=\varphi(\tau \pm 0), a(w, \tau \pm 0)=\psi(\tau \pm 0) .
$$

We also remark that the limits

$$
\lim _{\substack{r \rightarrow \infty \\ s \rightarrow \infty}} a(r \tau, s \sigma)=: a_{\infty}(\tau, \sigma)
$$

exist for almost every $(\tau, \sigma) \in \mathbb{T}^{2}$ and represent a function $a_{\infty} \in P C(\mathbb{T}) \otimes P C(\mathbb{T})$, and thus a function with well-defined limits $a(\tau \pm 0, \sigma \pm 0)$ at each point $(\tau, \sigma) \in \mathbb{T}^{2}$. 
Theorem 6.3. Let $a \in P C_{d} \otimes P C_{d}$ and $(\gamma, \delta) \in\{0,1\}^{2}$. For $(\tau, \mu) \in \mathbb{T} \times[0,1]$, define $a_{\tau, \mu}^{1}$ and $a_{\tau, \mu}^{2}$ in $P C_{d}$ by

$$
\begin{aligned}
& a_{\tau, \mu}^{1}(z)=(1-\mu) a(\tau-0, z)+\mu a(\tau+0, z), \\
& a_{\tau, \mu}^{2}(z)=(1-\mu) a(z, \tau-0)+\mu a(z, \tau+0) .
\end{aligned}
$$

Then $T^{\gamma, \delta}(a)$ is Fredholm if and only if the operators $T^{\delta}\left(a_{\tau, \mu}^{1}\right)$ and $T^{\gamma}\left(a_{\tau, \mu}^{2}\right)$ are invertible on $A^{2}(\mathbb{C}, d \mu)$ for all $(\tau, \mu) \in \mathbb{T} \times[0,1]$.

In the case of $N \geq 3$ dimensions, the Fredholmness of a mixed Toeplitz operator is equivalent to the invertibility of $N$ families of mixed Toeplitz operators on $A^{2}\left(\mathbb{C}^{N-1}, d \mu\right)$. For $\gamma=\delta=0$, the previous theorem is Duduchava's [15].

Theorem 6.4. Let $a \in P C_{d} \otimes P C_{d}$ and $(\gamma, \delta) \in\{0,1\}^{2}$.

(a) If the sequence

$$
T_{n}^{\gamma, \delta}(a):=\left(P_{n} \otimes P_{n}\right) T^{\gamma, \delta}\left(P_{n} \otimes P_{n}\right) \mid \operatorname{Im}\left(P_{n} \otimes P_{n}\right)
$$

is stable, then the four operators (36) are invertible and

$$
\lim _{n \rightarrow \infty}\left\|\left[T_{n}^{\gamma, \delta}(a)\right]^{-1}\right\|=\max _{(\alpha, \beta) \in\{0,1\}^{2}}\left\|\left[T^{\gamma \alpha, \delta \beta}\left(a^{\alpha, \beta}\right)\right]^{-1}\right\| .
$$

(b) For each $\varepsilon>0$,

$$
\lim _{n \rightarrow \infty} \Lambda_{\varepsilon}\left(T_{n}^{\gamma, \delta}(a)\right)=\bigcup_{(\alpha, \beta) \in\{0,1\}^{2}} \Lambda_{\varepsilon}\left(T^{\gamma \alpha, \delta \beta}\left(a^{\alpha, \beta}\right)\right) .
$$

We finally remark that Theorems 5.1 and 5.2 may also be "tensored": polynomial or analytical element collocation is applicable to $T^{1,1}(\mathrm{a})$ if and only if the four operators (35) are invertible.

\section{References}

[1] G. R. Allan, Ideals of vector-valued functions, Proc. London Math. Soc. (3) 18 (1968), 193-216.

[2] V. Bargmann, On a Hilbert space of analytic functions and an associated integral transform, Comm. Pure Appl. Math. 14 (1961), 187-214.

[3] C. A. Berger and L. A. Coburn, Toeplitz operators and quantum mechanics, J. Funct. Anal. 68 (1986), 273-299.

[4] -, 一, Toeplitz operators on the Segal-Bargmann space, Trans. Amer. Math. Soc. 301 (1987), 813-829.

[5] A. Böttcher, Truncated Toeplitz operators on the polydisk, Monatsh. Math. 110 (1990), 23-32.

[6] —, Pseudospectra and singular values of large convolution operators, J. Integral Equations Appl. 6 (1994), 267-301.

[7] A. Böttcher and B. Silbermann, The finite section method for Toeplitz operators on the quarter-plane with piecewise continuous symbols, Math. Nachr. 110 (1983), 279-291.

[8] —, 一, Analysis of Toeplitz operators, Springer, Berlin, 1990.

[9] A. Böttcher and H. Wolf, Finite sections of Segal-Bargmann space Toeplitz operators with polyradially continuous symbols, Bull. Amer. Math. Soc. (N.S.) 25 (1991), 365-372. 
[10] A. Böttcher, H. Wolf, Large sections of Bergman space Toeplitz operators with piecewise continuous symbols, Math. Nachr. 156 (1992), 129-155.

[11] -, -, Galerkin-Petrov methods for Bergman space Toeplitz operators, SIAM J. Numer. Anal. 30 (1993), 846-863.

[12] - - - Asymptotic invertibility of Bergman and Bargmann space Toeplitz operators, Asymptotic Anal. 8 (1994), 15-33.

[13] L. A. Coburn, Singular integral operators and Toeplitz operators on odd spheres, Indiana Univ. Math. J. 23 (1973), 433-439.

[14] R. G. Douglas, Banach Algebra Techniques in Operator Theory, Academic Press, New York, 1972

[15] R. V. Duduchava, Discrete convolution operators on the quarter-plane and their indices, Math. USSR-Izv. 11 (1977), 1072-1084.

[16] G. B. Folland, Harmonic Analysis in Phase Space, Princeton Univ. Press, Princeton, N.J., 1989.

[17] I. Gohberg and I. Feldman, Convolution Equations and Projection Methods for Their Solution, Amer. Math. Soc., Providence, R.I., 1974.

[18] I. Gohberg and N. Krupnik, On the algebra generated by Toeplitz matrices, Functional Anal. Appl. 3 (1969), 119-127.

[19] A. V. Kozak, On the reduction method for multidimensional discrete convolutions, Mat. Issled. 8 (1973), 157-160 (in Russian).

[20] G. McDonald, Toeplitz operators on the ball with piecewise continuous symbol, Illinois J. Math. 23 (1979), 286-294.

[21] L. Reichel and L. Trefethen, Eigenvalues and pseudo-eigenvalues of Toeplitz matrices, Linear Algebra Appl. 162 (1992), 153-185.

[22] P. Schmidt and F. Spitzer, The Toeplitz matrices of an arbitrary Laurent polynomial, Math. Scand. 8 (1960), 15-38.

[23] I. Segal, Lectures at the Summer Seminar on Applied Mathematics, Boulder, Col., 1960.

[24] B. Silbermann, Lokale Theorie des Reduktionsverfahrens für Toeplitzoperatoren, Math. Nachr. 104 (1981), 137-146.

[25] H. Widom, Singular integral equations in $L^{p}$, Trans. Amer. Math. Soc. 97 (1960), 131-160.

[26] - Asymptotic behavior of block Toeplitz matrices and determinants II, Adv. Math. 21 (1976), 1-29.

[27] - On the singular values of Toeplitz matrices, Z. Anal. Anwendungen 8 (1989), 221-229.

Added in proof. Our interest in $\varepsilon$-pseudospectra was excited by the paper [21], but it should be noted that the idea of the $\varepsilon$-pseudospectrum is much older. See, for example, Henry Landau's papers

H. Landau, Loss of unstable resonators, J. Opt. Soc. Amer. 66 (1976), 525-529;

- The notion of approximate eigenvalues applied to an integral equation of laser theory, Quart. Appl. Math. 35 (1977), 165-172.

For some recent developments related to spectral approximation, see also

W. Arveson, $C^{*}$-algebras and numerical linear algebra, J. Funct. Anal. 122 (1994), 333-360. S. Roch and B. Silbermann, $C^{*}$-algebra techniques in numerical analysis, J. Operator Theory 35 (1996), 241-280. 